\title{
Dynactin p150Glued-Deficiency in Midbrain Dopaminergic Neurons Leads to Progressive Neurodegeneration and Endoplasmic Reticulum Dysfunction
}

Jia Yu

Beijing University of Chinese Medicine

\section{Carmelo Sgobio}

Ludwig Maximilians University Munich: Ludwig-Maximilians-Universitat Munchen

\section{Xuan Yang}

Beijing University of Chinese Medicine

\section{Binbin Song}

Beijing University of Chinese Medicine

Jiayin Zheng

Beijing University of Chinese Medicine

Kuo Li

Beijing University of Chinese Medicine

\section{Lixin Sun}

National Institute on Aging

Huaibin Cai ( $\sim$ caih@mail.nih.gov)

National Institute on Aging https://orcid.org/0000-0002-8596-6108

\section{Research Article}

Keywords: Perry syndrome, dynactin p150Glued, GAP-Gly microtubule binding domain, midbrain dopaminergic neuron, neurodegeneration, unfolded protein response, ER stress

Posted Date: February 8th, 2022

DOI: https://doi.org/10.21203/rs.3.rs-1324919/v1

License: (c) (i) This work is licensed under a Creative Commons Attribution 4.0 International License.

Read Full License 


\section{Abstract \\ Background}

Multiple missense mutations in DCTN1 gene have been linked to Perry syndrome (PS), a rare neurodegenerative disease clinically manifested with parkinsonism, mental depression, and central hypoventilation. DCTN1 encodes dynactin $\mathrm{p} 150^{\text {Glued }}$ protein, the largest subunit of dynactin motor protein complex. The PS-related mutations are concentrated at the amino-terminal cytoskeleton-associated protein and glycine-rich (CAP-Gly) domain, which mediates the association of p150 Glued with microtubules. Degeneration of nigrostriatal dopaminergic neurons (DANs) was reported in the postmortem brains of PS patients. However, how the dysfunction of $\mathrm{p} 150^{\text {Glued }}$ affects the function and survival of DANs remains to be determined.

\section{Methods}

Using Cre-LoxP genetic manipulation, we crossbred Dctn 1 knock-in (KI) mice with Th-Cre mice to generate Dctn 1 conditional knockout (cKO) mice for genetic deletion of $\mathrm{p} 150^{\text {Glued }}$ protein in catecholaminergic neurons, including midbrain DANs. We also crossbred the Dctn $1 \mathrm{KI}$ mice with Cre/ Esr1 mice to generate pups for primary culture of Dctn 1 cKO cells. We then performed series of biochemical, neurochemical, cell biology, behavioral and neuropathological studies on the Dctn 1 cKO mice or cells to investigate the underlying pathological mechanisms.

\section{Results}

While both Dctn 1 control and cKO mice showed deterioration of rotarod motor performance during aging, the process was greatly accelerated in the CKO mice. The loss of $\mathrm{p} 150^{\text {Glued }}$ led to progressive degeneration of axon fibers and then cell bodies of midbrain DANs. In addition to neurodegeneration, abnormal accumulation of a-synuclein was found in the soma and nuclei of DANs in aged cKO mice. Moreover, before the onset of axon atrophy, both dopamine transporter (DAT) levels and activity were reduced in the DAN axon terminals of cKO mice. Further studies revealed unusually large spheric structures in the axons and dendrites of cKO DANs. Interestingly, endoplasmic reticulum (ER) stress protein BiP was abnormally accumulated in the dendritic spheroids and soma of cKO DANs. Accordingly, the Dctn 1 cKO cells displayed impaired ER export and increased unfolded protein response (UPR). As a result, the Dctn 1 cKO DANs were more vulnerable to ER stress-induced cell death.

\section{Conclusion}

Our findings demonstrate that $\mathrm{p} 150^{\text {Glued }}$ is important in maintaining the function and survival of midbrain

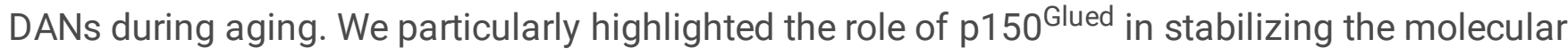


machinery for ER export, suggesting that the PS-related $\mathrm{p} 150^{\text {Glued }}$ dysfunction may render the midbrain DANs more susceptible to UPR and ER stress.

\section{Introduction}

Dynein and dynactin motor protein complex mediates the retrograde intracellular transport along microtubule networks in neurons [1]. Genetic mutations in genes encoding components of dynein and dynactin motor protein complex often cause deficits in axonal transport and neurological disorders [2]. A missense mutation in the largest dynactin subunit p150 Glued, encoded by gene, has been linked to a form of slowly progressive autosomal dominant form of lower motor neuron disease, the distal hereditary motor neuropathy 7B (HMN7B) [3]. The mutation, a single-base pair change (957C-T) in the second coding exon of DCTN1 gene, results in an amino acid substitution of G59S in the conserved, cytoskeletonassociated protein and glycine-rich (CAP-Gly) domain of $\mathrm{p} 150^{\text {Glued }}$ protein. Since then, a number of missense mutations (F52L, K56R, G67D, K68E, G71A/R/E/V, T72P, Q74P, Y78C/H, and Q93H) in the CAPGly domain of $\mathrm{p} 150^{\text {Glued }}$ have been identified as the genetic cause of Perry syndrome (PS) [4], a rare hereditary neurodegenerative disease characterized by parkinsonism with severe mental depression, weight loss, and central hypoventilation [5-7]. Dysfunction of dopamine transmission and degeneration of nigral DANs were reported in PS patients [7], which might contribute to the Parkinson's disease-like clinical symptoms. However, how these PS-related mutations affect the function and survival of nigrostriatal dopaminergic neurons (DANs) remains to be determined.

The PS-related missense mutations likely alter the conformation of CAP-Gly domain, which thereby interferes with the association of $\mathrm{p} 150^{\text {Glued }}$ protein with microtubules and other microtubule end-binding proteins [8], resulting in reduced processivity of dynein and dynactin complex with microtubes [9-15]. While genetic deletion of $\mathrm{p} 150^{\text {Glued in }}$ Dctn 1 homozygous knockout mice causes early embryonic lethality [11], the CAP-Gly domain of $\mathrm{p} 150^{\text {Glued }}$ seems dispensable for dynein/dynactin-mediated retrograde transport in postmitotic cells, like neurons. An alternative splicing variant, p135, which lacks the CAP-Gly domain of $\mathrm{p} 150^{\text {Glued }}$, may partially compensate for the loss of full-length $\mathrm{p} 150^{\text {Glued }}$ protein in retrograde transport $[16,17]$. These observations suggest the CAP-Gly domain of $\mathrm{p} 150^{\text {Glued }}$ may possess some distinct properties in maintaining the function and survival of selective neuron types implicated in PS and other degenerative neurological disorders $[7,15]$. In line with this notion, the spinal motor neurons appear to be more vulnerable to the loss of $\mathrm{p} 150^{\text {Glued }}$ protein than corticospinal motor neurons [17]. Therefore, it would be imperative to understand the selective vulnerability and underlying mechanisms of different neuronal types in PS. Like the homozygous Dctn 1 knockout (KO) mice [11], the homozygous G71A KI mice died embryonically [18], suggesting that the PS-related Dctn 1 mutation also leads to the dysfunction of $\mathrm{p} 150^{\text {Glued }}$ protein.

Various degrees of neuropathological abnormalities were identified in many different brain regions of PS patients [19]. In this study, we focused on investigating the functional significance of p150 Glued and its CAP-Gly domain in PS-related midbrain DAN degeneration using genetically engineered mouse models. 
Through crossbreeding Th-Cre transgenic mice [20] with Dctn 1 knock-in (KI) mice [17], we generated Dctn 1 conditional knockout (cKO) mice. The cKO mice lacked the CAP-Gly domain-containing p $150^{\text {Glued }}$ full-length protein in midbrain DANs. We then performed series of in vitro and in vivo experiments to identify any behavioral, neurochemical, neuropathological, cell biology, and biochemical abnormalities in the cKO mice. Our findings highlighted the importance of CAP-Gly domain in maintaining the ER transport in DANs.

\section{Methods}

\section{Animals}

Dctn ${ }^{\text {LoxP }} \mathrm{KI}$ mice with two LoxP sites in intron 1 and 4 of Dctn 1 were created as described previously [17]. Dctn $1^{\text {LoxP } K I ~ m i c e ~ w e r e ~ c r o s s b r e d ~ w i t h ~ T h-C r e ~[20] ~(M M R R C, ~ S t o c k ~ N u m b e r: ~ 029177-U C D) ~ o r ~ C r e / E s r 1 ~}$

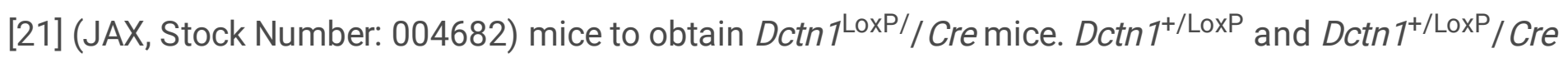
mice were further crossbred to generate $D c t n 1^{\text {LoxP/LoxP } / C r e ~ m i c e ~ i n ~ w h i c h ~ e x o n s ~} 2$ to 4 of $D c t n 1$ and thereby the GAP-Gly domain-containing $\mathrm{p} 150^{\text {Glued }}$ were deleted from the Cre-expressing cells. In this

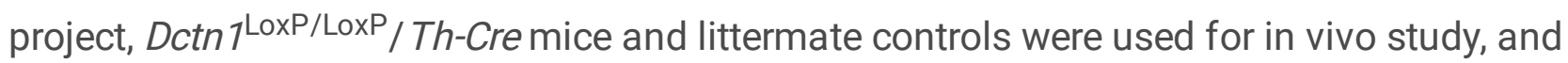
Dctn $7^{\text {LoxP/LoxP } / C r e / E s r 1 ~ p u p s ~ a n d ~ l i t t e r m a t e ~ c o n t r o l s ~ w e r e ~ u s e d ~ f o r ~ p r i m a r y ~ c e l l ~ c u l t u r e ~ a n d ~ f o r ~ i n ~ v i t r o ~}$ study. The mice were housed in a 12-hour light/dark cycle and fed regular diet ad libitum. All mouse work followed the guidelines approved by the Institutional Animal Care and Use Committees of the National Institute on Aging, NIH and Beijing Geriatric Hospital.

\section{Genotyping}

Animals were genotyped as described previously [17]. Genomic DNA was prepared from tail biopsy using DirectPCR Lysis Reagent (Viagen Biotech) and subjected to PCR amplification using specific sets of PCR

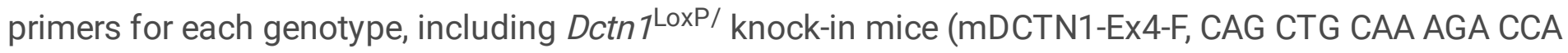
GCA AA; mDCTN1-Ex5-R: CAC ACC ACC TTC TTA GGC TTC A), Cre transgenic mice (CRE-F, CAT TTG GGC CAG CTA AAC AT; CRE-R, TGC ATG ATC TCC GGT ATT GA).

\section{Behavior tests}

Open-field test-As described previously [17, 22], male mice were placed in the open-field apparatus with infrared photobeam sensors. Locomotor activities (including ambulatory, rearing and fine movements) and time spent in the center area (around $40 \%$ of the total surface of the arena) of mice were measured by the Flex-Field Activity System (San Diego Instruments). Flex-Field software was used to trace and quantify mouse movement in the unit as the number of beam breaks per 30 minutes.

Rotarod test-As described previously [17, 23], male mice were placed onto a rotating rod with autoacceleration from $0 \mathrm{rpm}$ to $40 \mathrm{rpm}$ for $1 \mathrm{~min}$ (San Diego Instruments). The length of time the mouse stayed on the rotating rod was recorded. Three measurements were taken for each animal during each test. 


\section{Immunohistochemistry, light microscopy, and image analysis}

Mice were sacrificed and transcardially perfused with $4 \%$ paraformaldehyde (PFA) in cold phosphate buffered saline (PBS) as described previously $[17,23]$. Mouse brains were collected, post-fixed in $4 \%$ PFA/PBS solution overnight, submerged in $30 \%$ sucrose in PBS for at least 72 hours, and sectioned at 40 $\mu \mathrm{m}$ thickness using CM1950 cryostat (Leica). Frozen sections were stained with antibodies specific to p150 Glued (amino acid 3-202 at the N-terminus of p150 Glued, 1:200, BD Biosciences), p150 Glued/p135+ (amino acid 1266-1278 at the C-terminus of p150 Glued, 1:500, Abcam), tyrosine hydroxylase (TH, 1:2500, Pel-freez; 1:500, Sigma-Aldrich; 1:500, ImmunoStar; 1:500, Synaptic Systems), dopamine transporter (DAT, 1:500, Millipore), vesicular monoamine transporter 2 (VMAT2, 1:1000, Covance), glial fibrillary acidic protein (GFAP, 1:1000, Abcam), TAR DNA binding protein 43 (TDP-43, 1:500, Proteintech), a-synuclein (1:500, Santa Cruz), binding immunoglobulin protein (BiP, also referred to as GRP78, 1:500, Abcam, , and p-elF2a (Ser52) (1:500, Thermo Scientific) as suggested by manufacturers. Alexa Fluor 488-, 546- or 647conjugated secondary antibody (1:500, Invitrogen) was used to visualize the staining. Fluorescent images were captured using LSM 880 laser-scanning confocal microscope (Zeiss). The paired images in all the figures were collected at the same gain and offset settings. Post collection processing was applied uniformly to all paired images. The images were presented as either a single optic layer after acquisition in z-series stack scans at $1.0 \mu \mathrm{m}$ intervals from individual fields or displayed as maximum-intensity projections to represent confocal stacks. For the quantitative assessment of various marker protein distributions, images were taken using identical settings and exported to Image $(\mathrm{NIH})$ for imaging analysis. Images were converted to an 8-bit color scale (fluorescence intensity from 0 to 255) using ImageJ. The areas of interest were first selected with Polygon or Freehand selection tools and then subjected to measurement by mean optical intensities or area fractions. The mean intensity for the background area was subtracted from the selected area to determine the net mean intensity.

\section{Stereology}

Unbiased stereological estimation of midbrain dopaminergic neurons was performed as described previously $[24,25]$. According to the mouse brain in stereotaxic coordinates, series of $40-\mu \mathrm{m}$-thick coronal sections across the midbrain (every fourth section from Bregma -2.54 to $-4.24 \mathrm{~mm}$, ten sections per case) were stained with antibody specific to TH (1:2500, Pel-Freez) and subsequently visualized with Vectastain Elite ABC Kit and DAB Kit (Vector Laboratories). Bright field images were captured by Axio microscope Imager A1 (Zeiss). The number of TH-positive neurons was assessed using the optical fractionator function of Stereo Investigator 10 (MicroBrightField). Four or more mice were used per genotype at each time point. Counters were blinded to the genotypes of the samples. The sampling scheme was designed to have coefficient of error less than $10 \%$ in order to obtain reliable results.

\section{High-performance liquid chromatography (HPLC)}

HPLC for dopamine and DOPAC content in striatum was performed as described previously $[25,26]$. Mouse dorsal striatum was dissected, weighted and homogenized in $500 \mu$ l buffer $(0.1 \mathrm{~N}$ perchloric acid 
containing $100 \mu \mathrm{M}$ EDTA) per $100 \mathrm{mg}$ of tissue. After sonication and centrifugation, the supernatant was collected, frozen and stored at $-80^{\circ} \mathrm{C}$ until assayed for dopamine and 3,4-dihydroxyphenylacetic acid (DOPAC) by liquid chromatography with electrochemical detection. Briefly, mobile-phase solution containing octanesulfonic acid as an ion-pairing agent was pumped isocratically through a reversedphase liquid chromatographic column. Dopamine and DOPAC were quantified by the current produced after exposure of the eluate to a flow-through electrode set to oxidizing and then reducing potentials in series, with recordings from the last electrode reflecting reversibly oxidized species.

\section{Fast-scan cyclic voltammetry (FSCV)}

To investigate the kinetics of dopamine release evoked by electrical stimulation, FSCV was performed in $400-\mu \mathrm{m}$ slices of dorsal striatum as described previously $[25,27,28]$. Striatal slices were bathed in $32^{\circ} \mathrm{C}$ oxygenated artiđcial cerebrospinal पuid [aCSF: $126 \mathrm{mM} \mathrm{NaCl}, 2.5 \mathrm{mM} \mathrm{KCl}, 1.2 \mathrm{mM} \mathrm{NaH}_{2} \mathrm{PO}_{4}, 2.4 \mathrm{mM}$ $\mathrm{CaCl}_{2}, 1.2 \mathrm{mM} \mathrm{MgCl}_{2}, 25 \mathrm{mM} \mathrm{NaHCO}_{3}, 11 \mathrm{mM}$ glucose, $20 \mathrm{mM}$ 4-(2-hydroxyethyl)-1-

piperazineethanesulfonic acid, $0.4 \mathrm{mM}$ L-ascorbic acid]. Cylindrical carbon-fiber microelectrodes (50-100 $\mu \mathrm{m}$ of exposed fiber) were prepared with T650 fibers ( $6 \mu \mathrm{m}$ diameter, Goodfellow) and inserted into a glass pipette. The carbon-fiber electrode was held at $-0.4 \mathrm{~V}$, and the potential was increased to $1.2 \mathrm{~V}$ and back at $400 \mathrm{~V} / \mathrm{s}$ every $100 \mathrm{~ms}$ using a triangle waveform. Dopamine release was evoked by rectangular, electrical pulse stimulation (100-600 $\mu \mathrm{A}, 0.6 \mathrm{~ms}$ per phase, biphasic) applied every $5 \mathrm{~min}$. Data collection and analysis were performed using the Demon Voltammetry and Analysis software suite [29]. Ten cyclic voltammograms of charging currents were recorded as background before stimulation, and the average of these responses was subtracted from data collected during and after stimulation. Maximum amplitudes of extracellular dopamine transients were obtained from input/output function (I/O) curves. I/O curves were constructed by plotting stimulus current against the concentration of dopamine response amplitude over a range of stimulus intensities. The time constant of the slope decay $(\tau)$ was used for uptake kinetic analysis of evoked dopamine release. Following experiments, electrodes were calibrated using solutions of 1 and $10 \mu \mathrm{M}$ dopamine in aCSF. Cocaine hydrochloride and other chemicals used in FSCV were purchased from Sigma-Aldrich.

\section{Primary midbrain neuronal culture}

As described previously, mouse primary midbrain neuronal cultures were prepared from newborn Dctn ${ }^{\text {LoxP/LoxP } / C r e / E s r 1 ~ p u p s ~ a n d ~ l i t t e r m a t e ~ c o n t r o l s ~ o n ~ p o s t n a t a l ~ d a y ~} 0$ (P0) $[17,24]$. Briefly, midbrain tissues containing SNC and VTA were dissected and subjected to papain digestion $(5 \mathrm{U} / \mathrm{ml}$, Worthington Biochemicals) for $40 \mathrm{~min}$ at $37^{\circ} \mathrm{C}$. The digested tissue was carefully triturated into single cells using increasingly smaller pipette tips. The cells were then centrifuged at $250 \cdot \mathrm{g}$ for $5 \mathrm{~min}$ and resuspended in warm Basal Medium Eagle (BME, Sigma-Aldrich) supplemented with $5 \%$ heat-inactivated fetal bovine serum (FBS; Invitrogen), 1· N2/B27 supplement (100· stock, Invitrogen), 1· GlutaMax (100· stock, Invitrogen), $0.45 \%$ D-glucose (Sigma-Aldrich), $10 \mathrm{U} / \mathrm{ml}$ penicillin (Invitrogen), and $10 \mu \mathrm{g} / \mathrm{ml}$ streptomycin (Invitrogen). Dissociated midbrain neurons $\left(\sim 3 \times 10^{5}\right.$ cells per coverslip) were plated onto 12 -mm round coverslips precoated with poly-d-lysine and laminin (BD Bioscience) in 24-well plate, and maintained at $37^{\circ} \mathrm{C}$ in a $95 \% \mathrm{O}_{2}$ - and $5 \% \mathrm{CO}_{2}$-humidified incubator. Twenty-four hours after seeding, the cultures were 
switched to serum-free medium supplemented with $5 \mu \mathrm{M}$ cytosine $\beta$-D-arabinofuranoside (Sigma-Aldrich) to suppress the proliferation of glia and $1 \mu \mathrm{M}$ 4-hydroxytamoxifen (4-OHT, Sigma-Aldrich) to induce CRE recombinase activity. Starting from 5 days in vitro (DIV), culture medium was changed twice every week.

\section{Assessment of dopaminergic neuron survival after treatment with ER stress inducer}

The survival rate of dopaminergic neurons after treatment with ER stress inducer was assessed as described previously [24, 27]. 14 DIV primary midbrain neurons were exposed to 0 or $10 \mathrm{nM}$ thapsigargin (Sigma-Aldrich), an ER stress inducing compound. After 48 hours of treatment, neurons were fixed with 4\% PFA/PBS and immunostained with TH antibody and secondary antibody. The number of TH-positive neurons on each coverslip was counted under confocal microscope with a $\times 40$ objective. The survival rate of dopaminergic neurons was calculated by dividing the number of $\mathrm{TH}$-positive neurons on each coverslip by the number of TH-positive neurons on the control coverslip (neurons from Dctn $7^{\text {LoxP/LoxP }} \mathrm{PO}$ pups treated with $0 \mathrm{nM}$ thapsigargin).

\section{Primary fibroblast culture}

Mouse skin fibroblast cultures were prepared from the dorsal skin of newborn Dctn ${ }^{\text {LoxP/LoxP / Cre/Esr1 }}$ pups and littermate controls on postnatal day 0 (P0) as previously described [30]. Briefly, skin tissues were collected, rinsed in sterile PBS and minced into small pieces. The minced tissues were triturated with $2 \mathrm{ml}$ medium [DMEM (Invitrogen) supplemented with 10\% FBS, $10 \mathrm{U} / \mathrm{ml}$ penicillin, and $10 \mu \mathrm{g} / \mathrm{ml}$ streptomycin], sparsely plated into 10 -cm culture dish, and maintained at $37^{\circ} \mathrm{C}$ in a $95 \% \mathrm{O}_{2}$ and $5 \% \mathrm{CO}_{2}$ humidified incubator. After overnight (tissue fragments usually attach firmly to the dish), $8 \mathrm{ml}$ fresh medium was added. After 3-4 days (fibroblasts grown out of tissue fragments usually start to undergo rapid proliferation), medium was changed. On 7 DIV, fibroblasts were trypsinized with TrypLE (Invitrogen), passaged into new dishes, and treated with $1 \mu \mathrm{M}$ 4-hydroxytamoxifen (4-OHT) to induce CRE recombinase activity. From 11 DIV on, medium was changed every 3-4 days.

\section{Immunocytochemistry}

As described previously [24,31], cultured cells on coverslips were fixed with 4\% PFA in PBS for 15 minutes, permeabilized by $0.1 \%$ Triton X-100 for 5 minutes and blocked in $10 \%$ normal donkey serum (Invitrogen) in PBS for 1 hour at room temperature. Cells were then labeled with primary antibodies against $\mathrm{p} 150$ Glued (1:200, BD Biosciences), tyrosine hydroxylase (TH, 1:2000, Pel-freez; 1:500, SigmaAldrich; 1:500, ImmunoStar), microtubule-associated protein 2 (MAP2, 1:1000, Abcam), ERGIC53 (1:1000, Sigma-Aldrich), and a-tubulin (1:1000, Abcam) overnight at $4^{\circ} \mathrm{C}$ in a humidified chamber. After three washes with PBS, secondary antibodies conjugated to Alexa Fluor (Invitrogen; 1:1,000) were applied and incubated for 1 hour at room temperature in the dark. After extensive washes, coverslips were mounted on glass slides with Prolong diamond antifade reagent containing DAPI (Invitrogen), and fluorescence signals were detected using LSM 880 laser scanning confocal microscope (Zeiss). The paired images in 
all the figures were collected at the same acquisition settings, uniformly processed, presented as either a single optic layer or maximum-intensity projection of confocal stacks, and analyzed with ImageJ.

\section{Preparation of cell fractions}

Subcellular fractionation was performed using endoplasmic reticulum isolation kit (Sigma-Aldrich) according to the manufacturer's instruction. All procedures were performed at $4^{\circ} \mathrm{C}$. Mouse midbrains were isolated, cut into small pieces, and homogenized in four volumes of ice-cold Isotonic Extraction Buffer (10 mM HEPES, pH 7.8, 250 mM sucrose, 25 mM KCl, and 1 mM EGTA, Protease and Phosphatase Inhibitor Cocktail) with Dounce homogenizer (12 strokes). Cultured cells were harvested, washed with 10 volumes of PBS, and spun down at $600 \cdot g$ for 5 minutes. Cell pellet was suspended and incubated in 3 volumes of ice-cold Hypotonic Extraction Buffer (10 mM HEPES, pH 7.8, 25 mM KCl, and 1 mM EGTA, Protease and Phosphatase Inhibitor Cocktail) for 20 minutes to allow the cells to swell. Swollen cells were centrifuged at $600 \cdot g$ for 5 minutes. The new cell pellet was homogenized in 2 volumes of ice-cold Isotonic Extraction Buffer with Dounce homogenizer (10 strokes). The homogenate (referred to as total lysate) from brain tissues or cultured cells was spun at $1,000 \cdot g$ for 10 min. The supernatant (S1) was collected, and the pellet (P1) was saved as crude nuclei fraction. S1 was centrifuged at $12,000 \cdot g$ for 15 min. The supernatant (S2) was collected, and the pellet (P2) was saved as crude mitochondria fraction. S2 was further centrifuged at $100,000 \cdot \mathrm{g}$ for $60 \mathrm{~min}$. The supernatant (S3) was collected as cytosol fraction, and the pellet (P3) was saved as ER microsomes fraction. Crude nuclei fraction, crude mitochondria fraction and ER microsomes fraction were lysed in 1\% SDS buffer. SDS was added into the total lysate and cytosol fraction to $1 \%$ final concentration. Equal amount of total protein from total lysate and each fraction were resolved in SDS-PAGE and applied to western blot analysis.

\section{Western blot analysis}

Whole-cell lysates were prepared as described previously [32]. Cultured cells or mouse tissues were homogenized and sonicated in RIPA buffer (Sigma-Aldrich) or 1\% SDS lysis buffer (50 mM Tris- $\mathrm{HCl}, 150$ $\mathrm{mM} \mathrm{NaCl}, 2 \mathrm{mM}$ EDTA, pH 7.5, and 1\% SDS) supplemented with Protease Inhibitor Cocktails (Thermo Scientific). Lysates were clarified by centrifugation at $15000 \cdot \mathrm{g}$ for $15 \mathrm{~min}$ at $4^{\circ} \mathrm{C}$. The supernatants were quantified for protein content using the bicinchoninic acid (BCA) assay kit (Thermo Fisher Scientific). Equal amount of total proteins was separated by NuPage 4-12\% Bis-Tris gel (Thermo Scientific) using MES or MOPS running buffer (Thermo Scientific). The separated proteins were then transferred to nitrocellulose membranes using the Trans-Blot Turbo Transfer system (Bio-Rad) and incubated with specific primary antibodies. The antibodies used for western blot analysis included p $150^{\text {Glued }} \mathrm{N}$-terminus (specific to p150 Glued, 1:1000, BD Biosciences), p150 Glued C-terminus (specific to both p150 Glued and p135+, 1:1000, Abcam), dynactin subunit p62 (1:1000, Abcam), dynactin subunit p50 (1:1000, BD Biosciences), dynactin subunit actin related protein 1 (ARP1, 1:1000, Sigma-Aldrich), tyrosine hydroxylase (TH, 1:5000, Pel-freez; 1:1000, Sigma-Aldrich; 1:1000, ImmunoStar; 1:5000, Synaptic Systems), dopamine transporter (DAT, 1:1000, Millipore), vesicular monoamine transporter 2 (VMAT2, 1:5000, Covance), VAMP (vesicle-associated membrane protein)-associated protein B (VAPB, 1:1000, Proteintech), calnexin (1:1000, Proteintech), Sec13 [component of the coat protein complex II (COPII), 1:1000, Santa Cruz], 
Sec23 (component of COPII, 1:1000, Thermo Scientific), Sec31 (component of COPII, 1:1000, BD Biosciences), protein kinase-like endoplasmic reticulum kinase (PERK, 1:1000, Cell Signaling), p-PERK (Thr982) (1:1000, Abcam), eukaryotic initiation factor 2a (elF2a, 1:1000, Cell Signaling), p-elF2a (Ser52) (1:1000, Thermo Scientific), activating transcription factor 4 (ATF4, 1:1000, Proteintech), C/EBPhomologous protein (CHOP, 1:1000, Cell Signaling), inositol requiring protein 1a (IRE1a, 1:1000, Abcam), p-IRE1a (Ser724) (1:1000, Abcam), unspliced X-box binding protein 1 (unspliced XBP1, 1:1000, Proteintech), spliced XBP1 (1:1000, Proteintech), stress-activated protein kinase/Jun-amino-terminal kinase (SAPK/JNK, 1:1000, Cell Signaling), p-SAPK/JNK (Thr183/Tyr185) (1:1000, Cell Signaling), activating transcription factor (ATF6, 1:1000, Proteintech), cleaved Caspase 3 (1:1000, Cell Signaling), GAPDH (1:1000, Sigma-Adrich), a-tubulin (1:5000, Abcam), and $\beta$-actin (1:5000, Sigma-Aldrich). Protein signals were visualized by IRDye secondary antibodies and Odyssey system (LI-COR Biosciences) and quantified with NIH ImageJ software.

\section{Statistical analysis}

Statistical analysis was performed using GraphPad Prism 9 (GraphPad Software). Data were presented as mean \pm SEM. Statistical significance was determined by comparing means of different groups using unpaired t-test,one-way ANOVA with post-hoc Tukey test, two-way ANOVA with post-hoc Bonferroni test, or two-way ANOVA with Sidak's multiple comparisons test. ${ }^{\star} p<0.05,{ }^{\star \star} p<0.01,{ }^{\star \star \star} p<0.001,{ }^{\star \star \star *} p<0.0001$.

\section{Results}

\section{Generation ofDctn 1 conditional knockout mice that selectively delete $\mathrm{p} 150^{\text {Glued }}$ in midbrain dopaminergic neurons}

We crossbred Th-Cre mice [20] with $\operatorname{Dctn} 1$ LoxP $\left(D c t n 1^{+/ L o x P}\right) \mathrm{KI}$ mice [17] to generate $\operatorname{Dctn} 1^{+/+}[$referred as wild-type (WT)], Th-Cre/Dctn $1^{+/+}$(referred as Cre), Dctn ${ }^{\text {Loxp/LoxP }}$ [referred as control (Ctrl)], and ThCre/Dctn ${ }^{\text {LoxP/LoxP }}$ conditional knockout (referred as CKO) mice. The composition of offspring with different genotypes followed the Mendelian ratio, indicating a normal embryonic development of cKO mice. Western blot analyses revealed substantially reduced Dctn 1 full-length $\mathrm{p} 150^{\text {Glued }}$ protein, but markedly increased the levels of alternatively spliced p135 and related forms (p135+) in the midbrain tissues dissected from 1-month-old cKO mice compared to the age-matched WT, Cre and Ctrl mice (Fig. 1a, b). The Dctn1 p135+ forms lack the CAP-Gly domain [17]. By contrast, the levels of dynactin p62, p50, ARP1 subunits were not affected in the cKO mice (Fig. 1a). Additionally, the levels of p150 Glued and other dynactin components were comparable in the olfactory bulb, cerebral cortex, hippocampus, striatum, cerebellum and brainstem of Ctrl and cKO mice (Supplementary Fig. S1). The residual p150 Glued protein detected in the cKO samples is likely derived from the non-dopaminergic cells in the tissue preparations (Fig. 1a, b). Indeed, immunostaining of midbrain sections with an antibody against the CAPGly domain-containing N-terminal of $\mathrm{p} 150^{\text {Glued }}$ demonstrated a complete loss of $\mathrm{p} 150^{\text {Glued }}$ staining in the tyrosine hydrogenase (TH)-positive DANs (Fig. 1c). Therefore, we generated a line of Dctn $1 \mathrm{cKO}$ mice that disrupted the expression of $\mathrm{p} 150^{\text {Glued }}$ in midbrain DANs. 
In a parallel study, we crossbred the tamoxifen inducible Cre/Esr1 transgenic mice [21] with Dctn $1^{\text {Loxp } /+} \mathrm{KI}$ mice and isolated the midbrain tissues from postnatal day 1 (P1) pups for neuronal cultures. Western

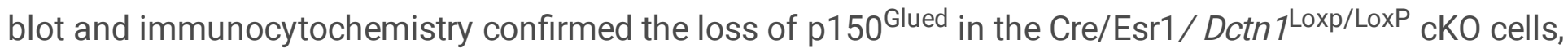
including the DANs (Supplementary Fig. S2). The neuronal cultures allowed for in-depth cell biology and biochemical studies on the role of $\mathrm{p} 150^{\text {Glued }}$ in intracellular transport.

\section{Dctn1cKO mice exhibit more profound deterioration of rotarod performance than the controls during aging}

The Dctn 1 cKO mice developed normally and displayed no overt behavior phenotypes. Although weight loss is a typical clinical manifestation of PS patients [7], the CKO mice weighted similarly as the littermate Ctrl mice at 1, 3, 6, 12, and 18 months of age (Fig. 2a). The cKO mice also displayed normal locomotion, rearing, fine movement, and time spent in the center of arena in the Open-field test when examined at 1,3 , 6,12 , and 18 months of age (Fig. $2 \mathrm{~b}-\mathrm{e}$ ). Since mouse models with deficiency in dopamine transmission are very sensitive to rotarod test [33,34], we also tested the performance of cKO mice in accelerating rotarod at different time points during aging. While the cKO mice performed as well as the Ctrl mice at 1 and 3 months of age in the rotarod test, the performance of cKO mice deteriorated more profoundly than the Ctrl mice starting at 6 months of age (Fig. 2f). Considering that the nigral DANs are essential in rotarod motor skill learning $[33,34]$, this accelerated deterioration of rotarod performance could be a consequence of the progressive loss of DANs and dysfunction of dopamine transmission in the Dctn 1 cKO mice during aging.

\section{Dctn1 cKO mice display progressive degeneration of DANs and other neuropathological abnormalities in the midbrain.}

Using unbiased stereological cell counting, we found that while the numbers of TH-positive midbrain DANs in substantia nigra pars compacta (SNc) and ventral tegmental area (VTA) were comparable between 12-month-old Ctrl and Dctn 1 cKO mice, the cell numbers were substantially reduced in the 24month-old Dctn 1 cKO mice compared to the age-matched Ctrl mice (Fig. 3a, b). Therefore, genetic deletion

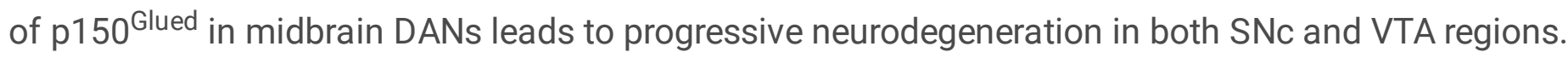

The dendrites of DANs located in the ventral SNc often protrude perpendicularly into the underneath substantia nigra par reticulata (SNr) region (Fig. 3c) and form synaptic connections with the incoming axon fibers [35]. Interestingly, TH staining revealed some remarkably large sphere-like structures often at the tips of DAN dendrites in the SNr of 6- and 24-month-old Dctn 1 cKO mice compared to the agematched Ctrl mice (Fig. 3c, d). The diameters of those dendritic spheroids were often larger than $20 \mathrm{~mm}$, and the numbers were substantially increased during the aging of Dctn $1 \mathrm{cKO}$ mice (Fig. 3c, d). In addition to the neuronal loss and dendritic morphological changes, increased astrogliosis visualized by glial fibrillary acidic protein (GFAP) staining was also observed in the SNc and SNr of 24-month-old Dctn 1 cKO mice (Supplementary Fig. 3a, b). Interestingly, a loss of nigral DANs and the appearance of sphere-like neurite swellings and gliosis were also observed in the substantia nigra (SN) and globus pallidus of PS 
patients $[6,19,36]$. Therefore, the Dctn 1 cKO mice recapitulate some key neuropathological features of PS.

\section{Abnormal somatic accumulation of a-synuclein but not TDP-43 in the midbrain DANs of agedDctn 1 cKO mice.}

Since abnormal TAR DNA-binding protein 43 (TDP-43)-positive cytoplasmic inclusions were often identified in the SN and other brain regions of PS patients $[6,19,36]$, we examined the subcellular location of TDP-43 in the midbrain DANs of 24-month-old Ctrl and Dctn 1 cKO mice. However, we did not detect any apparent accumulation of TDP-43 in the cytosol of either Ctrl or Dctn 1 cKO DANs (Fig. 4a, b). By contrast, although very sparse Lewy bodies were identified in the brain of PS patients [4,5], we observed a substantial increase of cytoplasmic and nuclear accumulation of a-synuclein in the midbrain DANs of 24-month-old Dctn 1 cKO mice compared to the Ctrl mice (Fig. 4c, d). While a-synuclein normally is enriched in the axon terminals, the abnormal cumulation of a-synuclein in cytosol and nucleus is implicated for neurodegeneration $[23,37,38]$.

\section{Progressive degeneration of DAN axon terminals in the dorsal striatum ofDctn1 cKO mice.}

As axon dystrophy was reported in the brains of PS patients $[6,19,36]$, we examined the density and morphology of DAN axon terminals in the dorsal striatum of Ctrl and Dctn1 cKO mice. We found a markedly reduction of axon density in the 24-month-old cKO mice compared to the age-matched controls (Fig. 5a, b). Furthermore, increasing numbers of abnormal axon swelling were observed in the 6- and 24month-old cKO mice compared to the age-matched controls (Fig. 5c, d). Interestingly, the swellings were packed with vesicular monoamine transporter 2 (VMAT2)-positive puncta (Fig. 5c), suggesting potential abnormalities in dopamine transmission in the dorsal striatum of Dctn1 cKO mice.

\section{Alterations of dopamine release and uptake in the dorsal striatum ofDctn $1 \mathrm{cKO}$ mice.}

Using HPLC, we did not detect any changes of dopamine (DA), dopamine metabolite 3,4dihydroxyphenylacetic acid (DOPAC), and 5-hydroxytryptamine (5-HT) content in the dorsal striatum of 12-month-old Dctn 1 cKO mice compared to controls (Fig. 6a). Correlatively, no apparent loss of DAN axon fibers was found in the same age group of Dctn1 cKO mice (Fig. 5b). We then quantified the evoked dopamine release by fast-scan cyclic voltammetry (FSCV) in striatal slices prepared from 12-month-old Dctn $1 \mathrm{Ctrl}$ and cKO mice. In response to either single- or burst $(25 \mathrm{~Hz})$-pulse stimulation, the peak evoked dopamine release was substantially higher from the cKO axon terminals compared to the controls (Fig. 6b, c). Additionally, the time constant of the slope delay, which measures the kinetics of dopamine uptake, was also increased in cKO axon terminals (Fig. 6d). Together, these data suggest that the loss of p150 Glued might reduce dopamine uptake at the Dctn $1 \mathrm{cKO}$ axon terminals, resulting in elevated dopamine concentration in the extrasynaptic space upon stimulation.

Reduced dopamine transporter expression and activity in the dorsal striatum of youngDctn1 1 KO mice. 
Since dopamine transporter (DAT) mediates the uptake of dopamine [39], we examined the protein levels of DAT, VMAT2, and TH in the striatal homogenates of 6-month-old Dctn $1 \mathrm{Ctrl}$ and cKO mice. The expression of DAT, but not VMAT2 or TH, was substantially reduced in the cKO samples (Fig. 7a, b). Immunostaining further confirmed a marked reduction of DAT-positive puncta in the dorsal striatum of cKO mice (Fig. 7c, d). In correlation with the reduced DAT expression, when pharmacologically blocked with DAT inhibitor cocaine, the increase of dopamine release was less robust from the cKO axon terminals compared to the controls (Fig. 7e). Together, these results demonstrate that the lack of p150 Glued leads to reduced expression and activity of DAT in DANs. Interestingly, reduced DAT ligand binding and activity were also observed in the striatum of PS patients [5].

\section{Abnormal accumulation of endoplasmic reticulum stress proteins in the dendritic spheroids and soma ofDctn1cKO midbrain dopaminergic neurons.}

To investigate the composition of neurite spheroids, we co-stained the midbrain sections with DAT, TH and various intracellular organelle markers and found that binding immunoglobulin protein (BiP), a residential protein in the lumen of endoplasmic reticulum (ER), was enriched in the dendritic spheroids of nigral DANs of 6- and 24-month-old Dctn 1 cKO mice (Fig. 8a, b). Furthermore, we also observed abnormal accumulation of BiP in the soma of cKO DANs during aging (Fig. 8c, d). BiP functions as molecular chaperon in protein folding, translocation, ER-associated degradation, and ER stress response [40, 41]. The abnormal accumulation of BiP in the soma and neurite of DANs indicates impairments of ER functions in the Dctn 1 cKO DANs.

\section{P150 Glued deficiency compromises ER export and protein maturation.}

A previous study demonstrates that $\mathrm{p} 150^{\text {Glued }}$ stabilizes the coat protein complex II (COPII) at ER exit site (ERES) through direct interaction with COPII component Sec23 $[42,43]$. Consistent with the early findings, we found that the levels of Sec23 and other COPII protein Sec13 and Sec31 were markedly reduced in the ER microsomes isolated from cultured Dctn 1 cKO fibroblasts (Fig. 9a, b). Furthermore, while the ERES is normally concentrated at one side of nucleus in the control fibroblasts as visualized by the ER-Golgi intermediate compartment $53 \mathrm{kDa}$ protein (ERGIC-53) immunostaining, the ERES was dispersed surrounding the nucleus of Dctn1 cKO fibroblasts (Fig. 9c). The reduction of Sec13 and Sec31 levels was also observed in the ER microsomes isolated from the midbrain of 4-month-old Dctn 7 LoxP/LoxP/CreEsr1

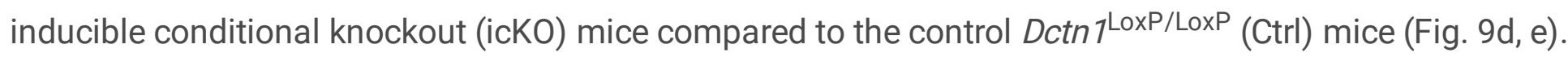
The concentration of COPII at ERES is critical for ER export of secretory and membrane proteins [44]. The relative levels of mature verse immature forms can be used as an indicator to estimate the efficiency of ER export in cells [31]. Accordingly, western blots showed substantial increase of immature DAT in the ER microsome fraction extracted from the midbrain of Dctn1 icKO mice compared to the controls (Fig. 9d, e),

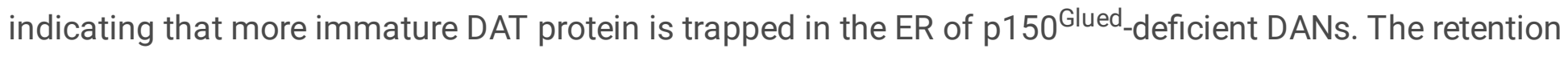
of immature DAT in ER may explain the reduction of DAT protein in the axon terminals of Dctn 1 cKO mice (Fig. 7). Therefore, our studies in Dctn 1 cKO fibroblasts and icKO midbrain tissues further support the 
notion that the lack of $\mathrm{p} 150^{\text {Glued }}$ destabilizes the COPII complex at ERES and compromises the ER export $[42,43]$.

\section{P150 Glued-deficiency activates unfolded protein response and exacerbates ER stress-induced dopaminergic neuron death.}

Since the accumulation of unfolded proteins in ER and deficiency in ER export trigger unfolded protein response (UPR) and ER stress [45], we examined the activation of a series of proteins implicated in the UPR pathway by western blot analyses. We found substantially increased phosphorylation of protein kinase R-like endoplasmic reticulum kinase (PERK), eukaryotic initiation factor-2a (elF2a), inositolrequiring enzyme $1 \mathrm{a}$ (IRE1a), and stress-activated protein kinase (SAPK) in the cKO cell lysates compared to the controls (Fig. 10a, b). We also observed upregulation of activating transcription factor 4 and 6 (AF4 and ATF6), CCAAT-enhancer-binding protein homologous protein (CHOP), spliced X-Box Binding Protein 1 (XBP1) and cleaved Caspase 3 in the cultured Dctn1 cKO fibroblasts (Fig. 10a, b). We further detected a markedly increase of phosphorylated elF2a (p- elF2a) in the midbrain DANs of 18month-old Dctn1 cKO mice (Fig. 10c, d). Finally, we found that the cultured Dctn1 cKO midbrain DANs were more susceptible to thapsigargin-induced ER stress and cell death (Fig. 10e). Together, these findings suggest an important function of $\mathrm{p} 150^{\text {Glued }}$ in protecting DANs against ER stress.

\section{Discussions}

In the present study we generated and characterized Dctn 1 cKO mice that genetically delete the CAP-Gly domain-containing $\mathrm{p} 150^{\text {Glued }}$ protein in midbrain DANs. Neuropathologically, the lack of $\mathrm{p} 150^{\text {Glued }}$ led to progressive loss of midbrain DANs, formation of large neurite spheroids, axon atrophy, and abnormal accumulation of a-synuclein in the soma and nuclei. Behaviorally, the cKO mice showed accelerated deterioration in rotarod motor performance compared to the control mice during aging. Furthermore, we found that the loss of $\mathrm{p} 150^{\text {Glued }}$ particularly affected the expression and subcellular localization of ERES proteins. The impairment of COPII-mediated ER export likely contributes to deficiency in ER-dependent protein maturation and export and increase of UPR and ER stress. Finally, we provide evidence that a lack of $150^{\text {Glued }}$ makes DANs more susceptible to ER stress, suggesting that ER stress may contribute to the loss of DANs in PS.

P150 Glued, the largest subunit of dynactin complex, exists as a dimer that binds directly to both microtubules and cytoplasmic dynein intermediate chain [13]. Previous in vitro studies demonstrate that p150 Glued is critical for movement of mitotic spindle and nucleus during cell proliferation [12]. Accordingly, we found that genetic deletion of $\mathrm{p} 150^{\text {Glued }}$ causes early embryonic lethality of Dctn 1 homozygous KO mice [11]. However, genetic deletion of $\mathrm{p} 150^{\text {Glued }}$ in postmitotic neurons does not lead to overt and widespread neuronal loss in mouse brains [17], supporting the notion that the cellular functions of p $150^{\text {Glued }}$ can be largely replaced with its CAP-Gly domain-lacking alternative splicing variants in neurons [16]. Nonetheless, since the HMN7B and PS-linked missense mutations all reside in the CAP-Gly 
domain of $\mathrm{p} 150^{\text {Glued }}[3,4]$, the CAP-Gly domain-containing $\mathrm{p} 150^{\text {Glued }}$ protein must participate in some distinct cellular processes important in maintaining the function and survival of spinal motor neurons and midbrain DANs during aging. Given that both Dctn 1 homozygous G59S and G71A KI mice show the same early embryonic lethality as the Dctn1 homozygous KO mice $[11,18]$, the HMN7B and PS-linked missense mutations must compromise the function of $\mathrm{p} 150^{\text {Glued }}$. Dynein/dynactin complex is critical for an array of cellular activities, including ER-to-Golgi transport, the centripetal movement of lysosomes and endosomes, spindle formation, chromosome movement, nuclear positioning, and axonogenesis [12]. Therefore, selective deletion of $\mathrm{p} 150^{\text {Glued }}$ in the disease-affected neuronal populations may provide a useful experimental platform to identify the key intracellular pathways responsible for their differential vulnerabilities in HMN7B and PS [17].

In a recent study, we genetically deleted $\mathrm{p} 150^{\text {Glued }}$ in the postnatal forebrain and spinal neurons, including

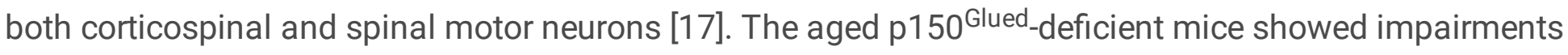
in rearing and rotarod tests, as well as a preferential degeneration of spinal motor neurons. Further histological and biochemical assays suggested that $\mathrm{p} 150^{\text {Glued }}$ may exert its protective function through regulating the transportation of autophagosomes, lysosomes, and postsynaptic glutamate receptors in spinal motor neurons. By contrast, when we genetically deleted $\mathrm{p} 150^{\text {Glued }}$ in midbrain DANs, we did not observe overt upregulations of autophagosome and lysosome markers (data not shown). Instead, we found that the lack of $\mathrm{p} 150^{\text {Glued }}$ particularly affected the ER function as documented with excessive accumulation of ER chaperone BiP in dendritic spheroids and soma, reduced levels of COPII components, deficiency in ER export, and increase of UPR and ER stress. These findings further support an important

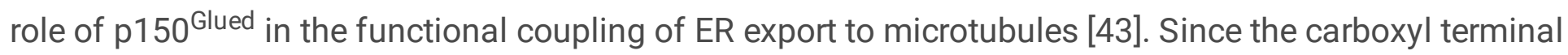
of DAT protein also binds to COPII component SEC24 and genetic knockdown of SEC24 impairs the cell surface targeting of DAT [46-48], the impaired ER export likely also contributes to the increased accumulation of immature DAT protein in the ER and reduced DAT level in the axon terminals of p150 Glued_deficient DANs.

While we focused the present study on the role of $\mathrm{p} 150^{\text {Glued }}$ in midbrain DANs and PS-related parkinsonism, it remains to be determined the underlying pathological mechanisms of PS-related psychiatric symptoms such as depression and apathy, which appeared as an early clinical feature prior to parkinsonism [4]. Except for the loss midbrain DANs, the postmortem brains of PS patients also displayed variable degrees of neurodegeneration in other nuclei in basal ganglia, as well as in hypothalamus, locus coeruleus (LC), periaqueductal gray matter, ventrolateral medulla, dorsal raphe nucleus, and pontine reticular formation [19]. Although the norepinephrine neurons in LC also express TH, the Th-Cre transgenic mice used in the present study showed a mosaic expression pattern of Cre DNA recombinase in the LC, in which around $30 \%$ of LC neurons contained Cre but no obvious loss of LC neurons was found (data not shown). Future studies are needed to crossbreed the Dctn $1 \mathrm{KI}$ mice with different Cre lines to continue exploring the role of $\mathrm{p} 150^{\text {Glued }}$ in other cell types. For example, the loss serotonergic neurons in the medullary raphe and ventrolateral medulla [49] may be responsible for the 
respiratory failure seen in PS patients. It would be interesting to test this hypothesis with a selective deletion of $\mathrm{p} 150^{\text {Glued }}$ in those serotonergic neurons using a suitable Cre line.

While the TDP-43-positive cytoplasmic inclusions were often identified in the SN and other brain regions of PS patients $[5,6,19,36]$, we did not detect any apparent accumulation of TDP-43 in the cytosol of Dctn 1 cKO DANs, suggesting that TDP-43 pathology might not contribute to the loss of midbrain DANs during aging. Nonetheless, future studies would be needed to determine whether the TDP-43 pathology contributes to the degeneration of other neuronal subtypes. In contrast, although the a-synucleincontaining Lewy bodies were rarely spotted in the postmortem brain of PS patients [4,5], we observed a substantial increase of cytoplasmic and nuclear accumulation of a-synuclein in the midbrain DANs of aged $D c t n 1$ cKO mice. Our findings are in line with previous studies which suggest that the abnormal somatic and nuclear cumulation of a-synuclein contributes to neurodegeneration [23, 37, 38]. However, it remains to determine how the lack of $p 150^{\text {Glued }}$ in the DANs leads to the retention of a-synuclein in cell bodies

\section{Conclusions}

To understand how the dysfunction of $\mathrm{p} 150^{\text {Glued }}$ protein contributes to PS-related parkinsonism and DAN loss, we genetically deleted $\mathrm{p} 150^{\text {Glued }}$ in the midbrain DANs of Dctn 1 cKO mice. The cKO mice recapitulated some key neuropathological features of PS, such as the degeneration of midbrain DANs, dysfunction of dopamine transporter DAT, axonal atrophy, appearance of large neurite spheroids and gliosis. We further revealed an important role of $\mathrm{p} 150^{\text {Glued }}$ in regulating COPII vesicles for ER export, suggesting that the lack of $\mathrm{p} 150^{\text {Glued }}$ may subject the midbrain DANs to UPR and ER stress-related cell death. Our studies raise the significance of ER stress in the degeneration of DANs. The Dctn 1 cKO mice may serve as a useful mouse model to further investigate the pathogenic mechanism of midbrain DAN loss in Parkinson's disease.

\section{Abbreviations}

HMN7B

hereditary motor neuropathy 7B

DCTN1

dynactin 1

CAP-Gly

cytoskeleton-associated protein and glycine-rich

PS

Perry syndrome

DAN

dopaminergic neuron

KO 
knockout

cKO

conditional knockout

$\mathrm{KI}$

knock-in

PFA

paraformaldehyde

DAT

dopamine transporter

$\mathrm{TH}$

tyrosine hydroxylase

VMAT2

vesicular monoamine transporter 2

GFAP

glial fibrillary acidic protein

TDP-43

TAR DNA binding protein 43

BiP

binding immunoglobulin protein

HPLC

high-performance liquid chromatography

DOPAC

3,4-dihydroxyphenylacetic acid

FSCV

fast-scan cyclic voltammetry

aCSF

artificial cerebrospinal fluid

BCA

bicinchoninic acid

VAPB

VAMP (vesicle-associated membrane protein)-associated protein B COPII

component of the coat protein complex II

Sec13, 23, and 31

components of COPII

PERK

protein kinase-like endoplasmic reticulum kinase

elF2a

eukaryotic initiation factor $2 a$

p-elF2a 
phosphorylated elF2a

ATF4 and 6

activating transcription factor 4 and 6

CHOP

CCAAT-enhancer-binding protein homologous protein

IRE1a

inositol requiring protein $1 \mathrm{a}$

XBP1

X-box binding protein 1

SPARK/JNK

stress-activated protein kinase/Jun-amino-terminal kinase

Ctrl

control

$\mathrm{SNc}$

substantia nigra pars compacta

VTA

ventral tegmental area

$\mathrm{SNr}$

substantia nigra par reticulata

$5-\mathrm{HT}$

5-hydroxytryptamine

ER

endoplasmic reticulum

ERES

ER exit site

ERGIC-53

ER-Golgi intermediate compartment $53 \mathrm{kDa}$ protein

UPR

unfolded protein response

LC

locus coeruleus

Declarations

\section{Ethics approval}

All animal procedures conformed to the $\mathrm{NIH}$ guide for the ethical care and use of laboratory animals.

Animal protocols were approved by the Institutional Animal Care and Use Committee of National Institute on Aging and Beijing Geriatric Hospital. 
All authors have read the manuscript and indicated consent for publication.

\section{Availability of data and materials}

All reagents and animal models will be available upon request.

\section{Competing interests}

The authors declare that they have no competing interests.

\section{Funding}

This work was supported in part by the intramural research programs of National Institute on Aging (AG000946), National Natural Science Foundation of China (No. 82071438 and 81601117), Beijing Natural Science Foundation (No. 7202077 and 7152077), Beijing BaiQianWan Talents Project (No. 2017002), and Beijing Nova Program (No. xx2018099).

\section{Authors' contributions}

$\mathrm{HC}$, JY, and CS conceived the research and designed the experiments. JY, CS, XY, BS, JZ, KL, and LS performed experiments. HC, CS and JY analyzed data and wrote manuscript. All authors read and approved the final manuscript.

\section{Acknowledgments}

The authors thank Dr. David M Lovinger of National Institute on Alcohol Abuse and Alcoholism for assisting the FSCV recording, members of Cai and Yu Labs for various supports, China Scholarship Council (CSC) for its International Exchange Program, and Beijing Hospital Authority for its Science \& Technology Innovation Program and High-level Talents Program.

\section{References}

1. Guzik BW, Goldstein LS: Microtubuledependent transport in neurons: steps towards an understanding of regulation, function and dysfunction. Current opinion in cell biology 2004, 16(4):443-450.

2. Levy JR, Holzbaur EL: Cytoplasmic dynein/dynactin function and dysfunction in motor neurons. International journal of developmental neuroscience : the official journal of the International Society for Developmental Neuroscience 2006, 24(2-3):103-111.

3. Puls I, Jonnakuty C, LaMonte BH, Holzbaur EL, Tokito M, Mann E, Floeter MK, Bidus K, Drayna D, Oh SJ et al: Mutant dynactin in motor neuron disease. Nature genetics 2003, 33(4):455-456.

4. Perry TL, Bratty PJ, Hansen S, Kennedy J, Urquhart N, Dolman CL: Hereditary mental depression and Parkinsonism with taurine deficiency. Arch Neurol 1975, 32(2):108-113. 
5. Konno T, Ross OA, Teive HAG, Slawek J, Dickson DW, Wszolek ZK: DCTN1-related neurodegeneration: Perry syndrome and beyond. Parkinsonism Relat Disord 2017, 41:14-24.

6. Farrer MJ, Hulihan MM, Kachergus JM, Dachsel JC, Stoessl AJ, Grantier LL, Calne S, Calne DB, Lechevalier B, Chapon F et al: DCTN1 mutations in Perry syndrome. Nature genetics 2009, 41(2):163165.

7. Dulski J, Cerquera-Cleves C, Milanowski L, Kidd A, Sitek EJ, Strongosky A, Vanegas Monroy AM, Dickson DW, Ross OA, Pentela-Nowicka J et al: Clinical, pathological and genetic characteristics of Perry disease-new cases and literature review. Eur J Neuro/ 2021.

8. Ligon LA, Shelly SS, Tokito M, Holzbaur EL: The microtubule plus-end proteins EB1 and dynactin have differential effects on microtubule polymerization. Mol Biol Cell 2003, 14(4):1405-1417.

9. Moughamian AJ, Holzbaur EL: Dynactin is required for transport initiation from the distal axon. Neuron 2012, 74(2):331-343.

10. Ayloo S, Lazarus JE, Dodda A, Tokito M, Ostap EM, Holzbaur EL: Dynactin functions as both a dynamic tether and brake during dynein-driven motility. Nat Commun 2014, 5:4807.

11. Lai C, Lin X, Chandran J, Shim H, Yang WJ, Cai H: The G59S mutation in p150(glued) causes dysfunction of dynactin in mice. The Journal of neuroscience : the official journal of the Society for Neuroscience 2007, 27(51):13982-13990.

12. Schroer TA: Dynactin. Annual review of cell and developmental biology 2004, 20:759-779.

13. Waterman-Storer CM, Karki S, Holzbaur EL: The p150Glued component of the dynactin complex binds to both microtubules and the actin-related protein centractin (Arp-1). Proc Natl Acad Sci U S A 1995, 92(5):1634-1638.

14. Li S, Finley J, Liu ZJ, Qiu SH, Chen H, Luan CH, Carson M, Tsao J, Johnson D, Lin G et al: Crystal structure of the cytoskeleton-associated protein glycinerich (CAP-Gly) domain. J Biol Chem 2002, 277(50):48596-48601.

15. Zhang J, Wang H, Liu W, Wang J, Zhang J, Chang X, Huang S, Pang X, Guo J, Wang Q et al: A novel Q93H missense mutation in DCTN1 caused distal hereditary motor neuropathy type 7B and Perry syndrome from a Chinese family. Neurol Sci 2021, 42(9):3695-3705.

16. Dixit R, Levy JR, Tokito M, Ligon LA, Holzbaur EL: Regulation of dynactin through the differential expression of p150Glued isoforms. The Journal of biological chemistry 2008, 283(48):33611-33619.

17. Yu J, Lai C, Shim H, Xie C, Sun L, Long CX, Ding J, Li Y, Cai H: Genetic ablation of dynactin p150(Glued) in postnatal neurons causes preferential degeneration of spinal motor neurons in aged mice. Mol Neurodegener 2018, 13(1):10.

18. Deshimaru M, Mishima T, Watanabe T, Kubota K, Hosoi M, Kinoshita-Kawada M, Yuasa-Kawada J, Ikeda M, Mori M, Murata $Y$ et al: Behavioral profile in a Dctn1(G71A) knock-in mouse model of Perry disease. Neurosci Lett 2021, 764:136234.

19. Wider C, Dickson DW, Stoessl AJ, Tsuboi Y, Chapon F, Gutmann L, Lechevalier B, Calne DB, Personett DA, Hulihan M et al: Pallidonigral TDP-43 pathology in Perry syndrome. Parkinsonism Relat Disord 2009, 15(4):281-286. 
20. Gong S, Doughty M, Harbaugh CR, Cummins A, Hatten ME, Heintz N, Gerfen CR: Targeting Cre recombinase to specific neuron populations with bacterial artificial chromosome constructs. $J$ Neurosci 2007, 27(37):9817-9823.

21. Hayashi S, McMahon AP: Efficient recombination in diverse tissues by a tamoxifen-inducible form of Cre: a tool for temporally regulated gene activation/inactivation in the mouse. Dev Biol 2002, 244(2):305-318.

22. Parisiadou L, Yu J, Sgobio C, Xie C, Liu G, Sun L, Gu XL, Lin X, Crowley NA, Lovinger DM et al: LRRK2 regulates synaptogenesis and dopamine receptor activation through modulation of PKA activity. Nature neuroscience 2014.

23. Lin X, Parisiadou L, Sgobio C, Liu G, Yu J, Sun L, Shim H, Gu XL, Luo J, Long CX et al: Conditional expression of Parkinson's disease-related mutant alpha-synuclein in the midbrain dopaminergic neurons causes progressive neurodegeneration and degradation of transcription factor nuclear receptor related 1. The Journal of neuroscience : the official journal of the Society for Neuroscience 2012, 32(27):9248-9264.

24. Liu G, Yu J, Ding J, Xie C, Sun L, Rudenko I, Zheng W, Sastry N, Luo J, Rudow G et al: Aldehyde dehydrogenase 1 defines and protects a nigrostriatal dopaminergic neuron subpopulation. The Journal of clinical investigation 2014, 124(7):3032-3046.

25. Liu G, Sgobio C, Gu X, Sun L, Lin X, Yu J, Parisiadou L, Xie C, Sastry N, Ding J et al: Selective expression of Parkinson's disease-related Leucine-rich repeat kinase 2 G2019S missense mutation in midbrain dopaminergic neurons impairs dopamine release and dopaminergic gene expression. Hum Mol Genet 2015, 24(18):5299-5312.

26. Holmes C, Eisenhofer G, Goldstein DS: Improved assay for plasma dihydroxyphenylacetic acid and other catechols using high-performance liquid chromatography with electrochemical detection. $J$ Chromatogr B Biomed App/ 1994, 653(2):131-138.

27. Lin X, Parisiadou L, Sgobio C, Liu G, Yu J, Sun L, Shim H, Gu X-L, Luo J, Long C-X et al: Conditional Expression of Parkinson's Disease-Related Mutant alpha-Synuclein in the Midbrain Dopaminergic Neurons Causes Progressive Neurodegeneration and Degradation of Transcription Factor Nuclear Receptor Related 1. Journal of Neuroscience 2012, 32(27):9248-9264.

28. Sgobio C, Sun L, Ding J, Herms J, Lovinger DM, Cai H: Unbalanced calcium channel activity underlies selective vulnerability of nigrostriatal dopaminergic terminals in Parkinsonian mice. Sci Rep 2019, 9(1):4857.

29. Yorgason JT, Espana RA, Jones SR: Demon voltammetry and analysis software: analysis of cocaineinduced alterations in dopamine signaling using multiple kinetic measures. $J$ Neurosci Methods 2011, 202(2):158-164.

30. Cho HJ, Liu G, Jin SM, Parisiadou L, Xie C, Yu J, Sun L, Ma B, Ding J, Vancraenenbroeck R et al: MicroRNA-205 regulates the expression of Parkinson's disease-related leucine-rich repeat kinase 2 protein. Human molecular genetics 2013, 22(3):608-620. 
31. Cho HJ, Yu J, Xie C, Rudrabhatla P, Chen X, Wu J, Parisiadou L, Liu G, Sun L, Ma B et al: Leucine-rich repeat kinase 2 regulates Sec16A at ER exit sites to allow ER-Golgi export. The EMBO journal 2014.

32. Luo J, Sun L, Lin X, Liu G, Yu J, Parisiadou L, Xie C, Ding J, Cai H: A calcineurin- and NFAT-dependent pathway is involved in alpha-synuclein-induced degeneration of midbrain dopaminergic neurons. Human molecular genetics 2014.

33. Wu J, Kung J, Dong J, Chang L, Xie C, Habib A, Hawes S, Yang N, Chen V, Liu Z et al: Distinct Connectivity and Functionality of Aldehyde Dehydrogenase 1a1-Positive Nigrostriatal Dopaminergic Neurons in Motor Learning. Cell Rep 2019, 28(5):1167-1181 e1167.

34. Beeler JA, Cao ZF, Kheirbek MA, Ding Y, Koranda J, Murakami M, Kang UJ, Zhuang X: Dopaminedependent motor learning: insight into levodopa's long-duration response. Ann Neurol 2010, 67(5):639-647.

35. Crittenden JR, Tillberg PW, Riad MH, Shima Y, Gerfen CR, Curry J, Housman DE, Nelson SB, Boyden ES, Graybiel AM: Striosome-dendron bouquets highlight a unique striatonigral circuit targeting dopamine-containing neurons. Proc Natl Acad Sci U S A 2016, 113(40):11318-11323.

36. Wider C, Dachsel JC, Farrer MJ, Dickson DW, Tsuboi Y, Wszolek ZK: Elucidating the genetics and pathology of Perry syndrome. J Neurol Sci 2010, 289(1-2):149-154.

37. Cooper AA, Gitler AD, Cashikar A, Haynes CM, Hill KJ, Bhullar B, Liu K, Xu K, Strathearn KE, Liu F et al: Alpha-synuclein blocks ER-Golgi traffic and Rab1 rescues neuron loss in Parkinson's models. Science 2006, 313(5785):324-328.

38. Kontopoulos E, Parvin JD, Feany MB: Alpha-synuclein acts in the nucleus to inhibit histone acetylation and promote neurotoxicity. Hum Mol Genet 2006, 15(20):3012-3023.

39. Sulzer D, Cragg SJ, Rice ME: Striatal dopamine neurotransmission: regulation of release and uptake. Basal Ganglia 2016, 6(3):123-148.

40. Stolz A, Wolf DH: Endoplasmic reticulum associated protein degradation: a chaperone assisted journey to hell. Biochim Biophys Acta 2010, 1803(6):694-705.

41. Mayer MP, Bukau B: Hsp70 chaperones: cellular functions and molecular mechanism. Cell Mol Life Sci 2005, 62(6):670-684.

42. Verissimo F, Halavatyi A, Pepperkok R, Weiss M: A microtubule-independent role of p150glued in secretory cargo concentration at endoplasmic reticulum exit sites. J Cell Sci 2015, 128(22):41604170.

43. Watson P, Forster R, Palmer KJ, Pepperkok R, Stephens DJ: Coupling of ER exit to microtubules through direct interaction of COPII with dynactin. Nature cell biology 2005, 7(1):48-55.

44. Dries DR, Yu G: Assembly, maturation, and trafficking of the gamma-secretase complex in Alzheimer's disease. Curr Alzheimer Res 2008, 5(2):132-146.

45. Hetz C, Papa FR: The Unfolded Protein Response and Cell Fate Control. Mol Cell 2018, 69(2):169-181.

46. Sucic S, El-Kasaby A, Kudlacek O, Sarker S, Sitte HH, Marin P, Freissmuth M: The serotonin transporter is an exclusive client of the coat protein complex II (COPII) component SEC24C. J Biol Chem 2011, 
286(18):16482-16490.

47. Bu M, Farrer MJ, Khoshbouei $\mathrm{H}$ : Dynamic control of the dopamine transporter in neurotransmission and homeostasis. NPJ Parkinsons Dis 2021, 7(1):22.

48. Oaks AW, Marsh-Armstrong N, Jones JM, Credle JJ, Sidhu A: Synucleins antagonize endoplasmic reticulum function to modulate dopamine transporter trafficking. PLOS One 2013, 8(8):e70872.

49. Tsuboi Y, Dickson DW, Nabeshima K, Schmeichel AM, Wszolek ZK, Yamada T, Benarroch EE:

Neurodegeneration involving putative respiratory neurons in Perry syndrome. Acta Neuropathol 2008, 115(2):263-268.

\section{Figures}

a

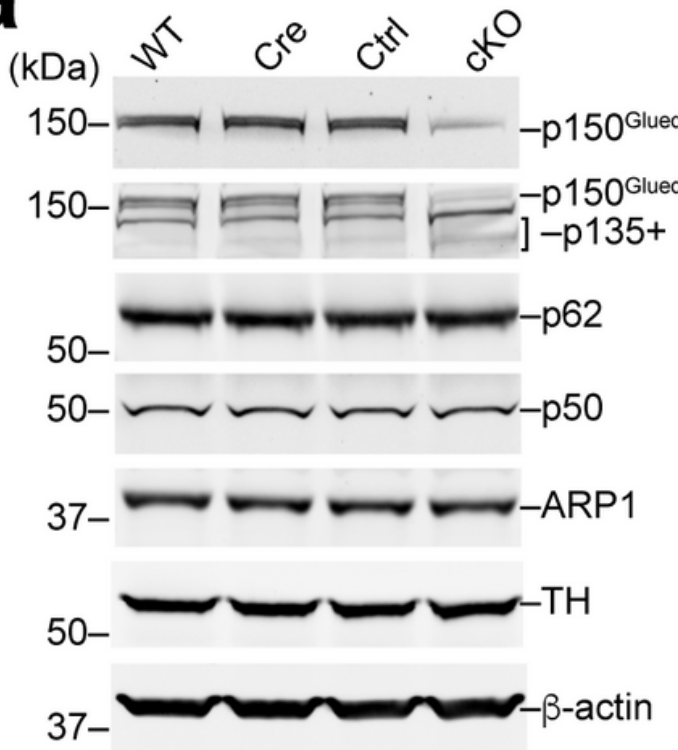

b

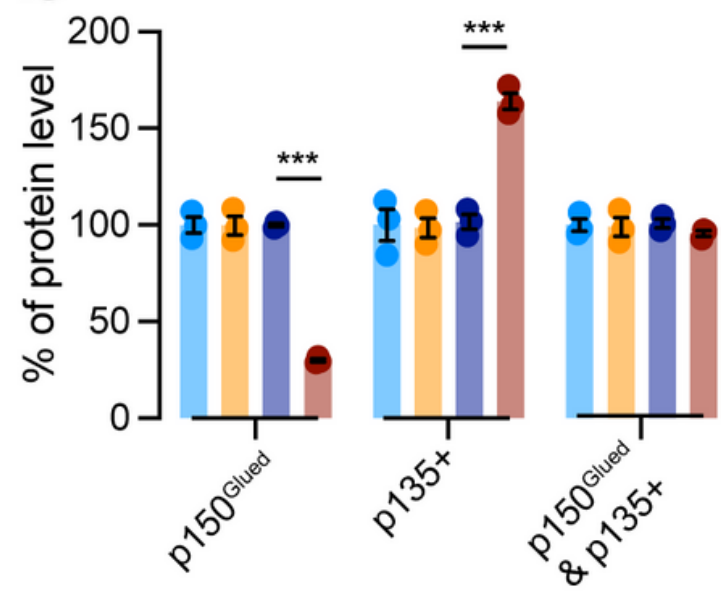

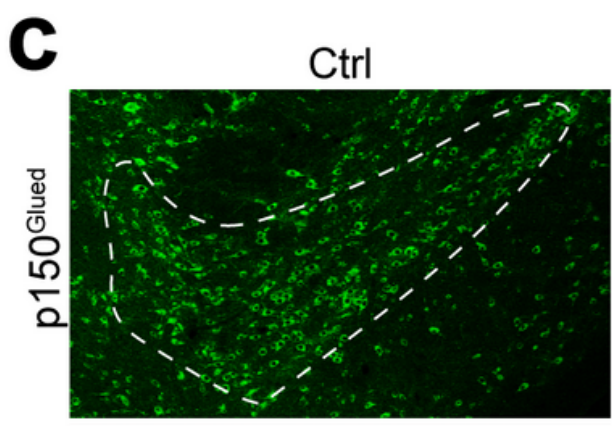
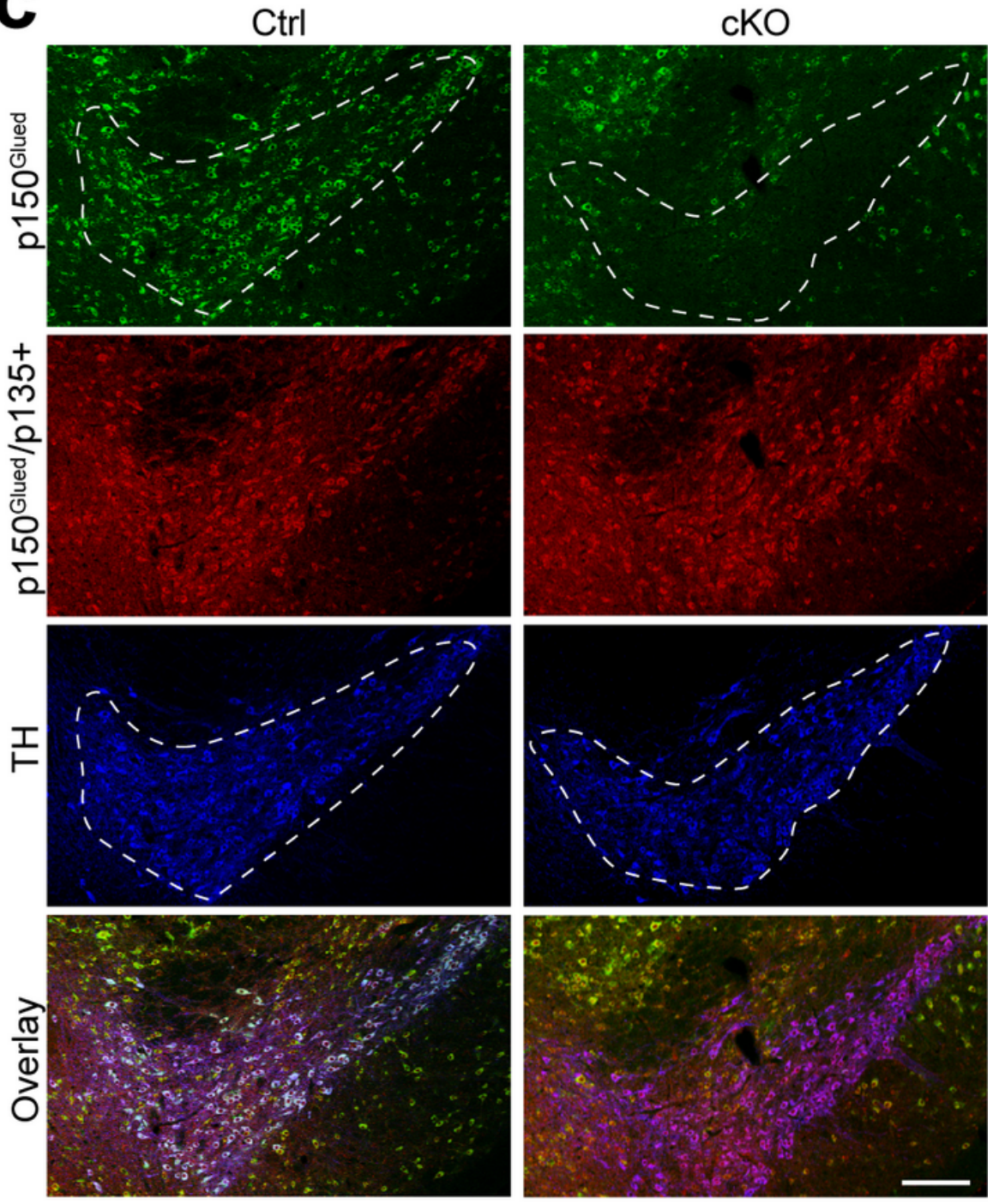

Figure 1 
Genetic deletion of $\mathbf{p} 150^{\text {Glued }}$ in midbrain dopaminergic neurons of cKO mice. (a) Western blots show the expression of p150 Glued, p135+, p62, p50, ARP1 and TH in the midbrain homogenates of 1-month-old WT, Cre, Ctrl, and cKO mice. b-actin and TH were used as loading control. (a) Bar graph estimates the levels of $\mathrm{p} 150^{\text {Glued }}, \mathrm{p} 135+$ and $\mathrm{p} 150^{\text {Glued }} / \mathrm{p} 135+$ proteins normalized with $\mathrm{b}$-actin $(\mathrm{n}=3$ per genotype). Data were presented as mean \pm SEM. One-way ANOVA plus Tukey's post hoc test was used for statistical analysis. ${ }^{\star \star \star} p<0.001$ for difference against cKO samples, while no difference was found among WT, Cre, and Ctrl samples. (c) Immunofluorescent images show the staining of p150 Glued (green), p150 Glued/p135+ (red) and TH (blue) in the midbrain coronal sections of 1-month-old Ctrl and cKO mice. DANs were visualized by TH staining. Scale bar: $250 \mu \mathrm{m}$. 

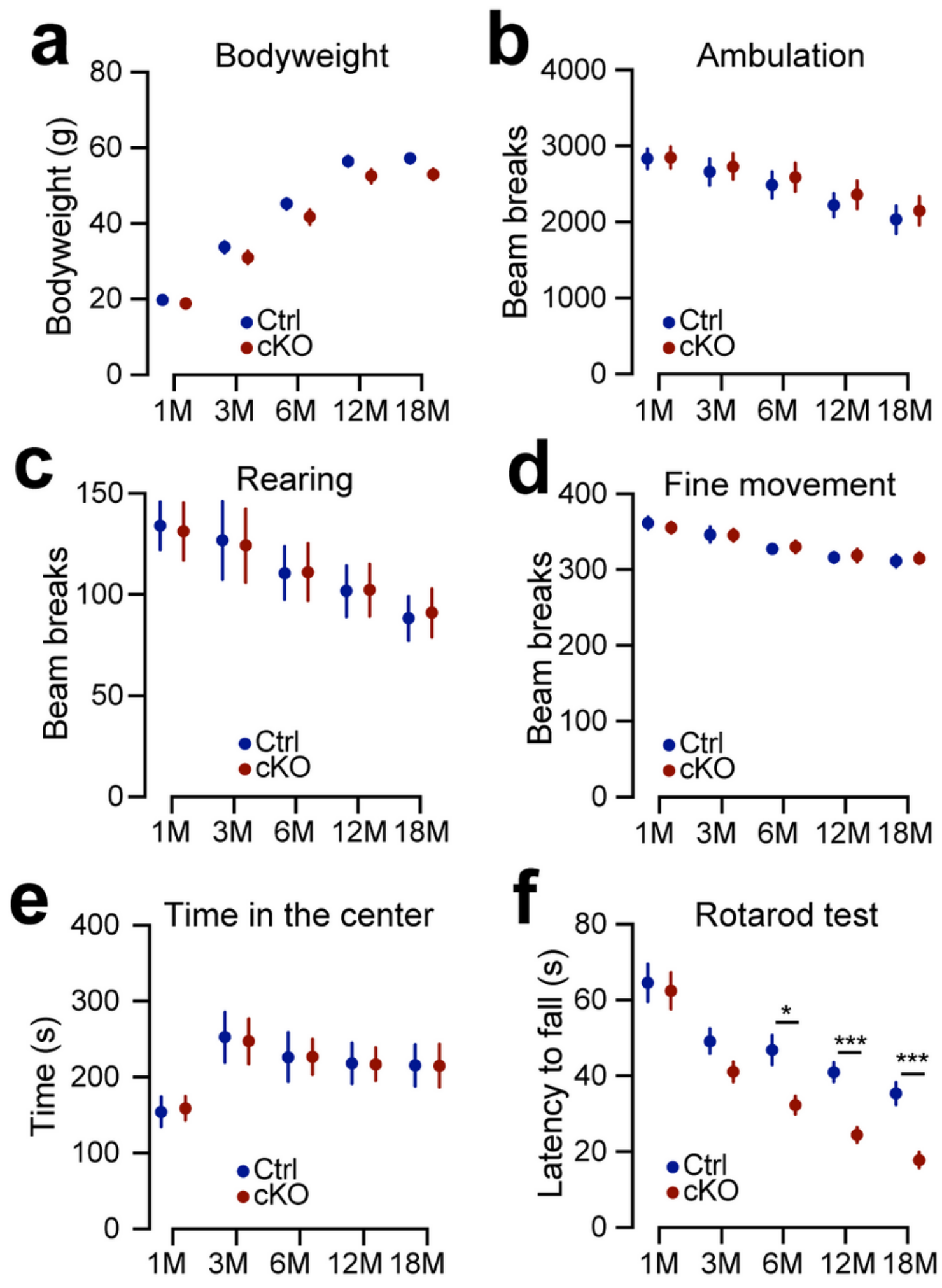

Figure 2

Accelerated deterioration of motor coordination in cKO mice. (a-f) At 1, 3, 6, 12 and 18 months of age, a cohort of male Ctrl and cKO mice ( $\mathrm{n}=16$ animals in each genotype) were repeatedly weighted (a); tested for ambulatory movement (b), rearing (c), fine movement (d) and center time (e) in open-field test; and examined for the latency to fall in rotarod test $(f)$. Data were presented as mean \pm SEM. Two-way ANOVA 
with Sidak's multiple comparisons test was used for statistical analysis. (f) In rotarod test, ${ }^{*} p=0.0178$ (6M), ${ }^{* \star *} p=0.0001$ (12M), ${ }^{* \star *} p=0.0002$ (18M).

a

b
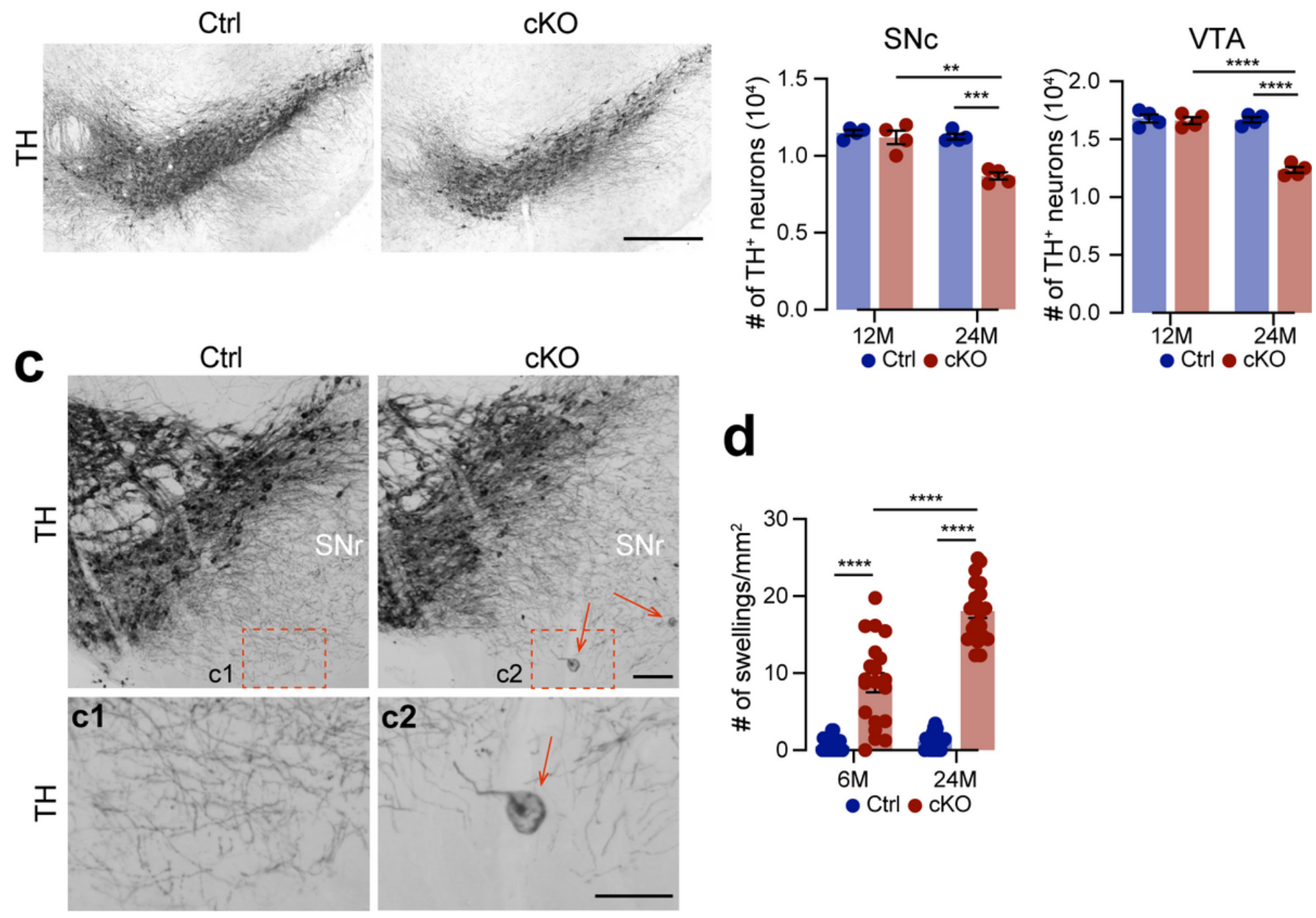

\section{Figure 3}

Progressive degeneration of dopaminergic neurons in the midbrain of cKO mice. (a)

Immunohistochemical images show TH staining in the midbrain coronal sections of 24-month-old Ctrl and cKO mice. Scale bar: $400 \mathrm{~mm}$. (b) Bar graphs depict unbiased stereological estimation of the number of TH-positive DANs in SNc and VTA of 12- and 24-month-old Ctrl and cKO mice ( $\mathrm{n}=4$ per genotype). Data were presented as mean \pm SEM. Unpaired t-test, ${ }^{* \star} p=0.0025,{ }^{* \star *} p=0.0001,{ }^{* \star \star *} p<0.0001$. (c) Representative images show TH staining in the SNc and SNr of 6-month-old Ctrl and cKO mice. Scale bar: top panel $500 \mathrm{~mm}$, bottom panel, $250 \mathrm{~mm}$. Arrows point to the abnormal, TH-positive dendritic spheroids in the SNr of cKO mice. (d) Bar graph quantifies the appearance of dendritic spheroids in SNr of 6-and 24-month-old Ctrl and cKO mice ( $\mathrm{n}=4$ animals per genotype and 5 sections per animal). Data were presented as mean \pm SEM. Unpaired t-test, ${ }^{\star \star \star \star} p<0.0001$. 

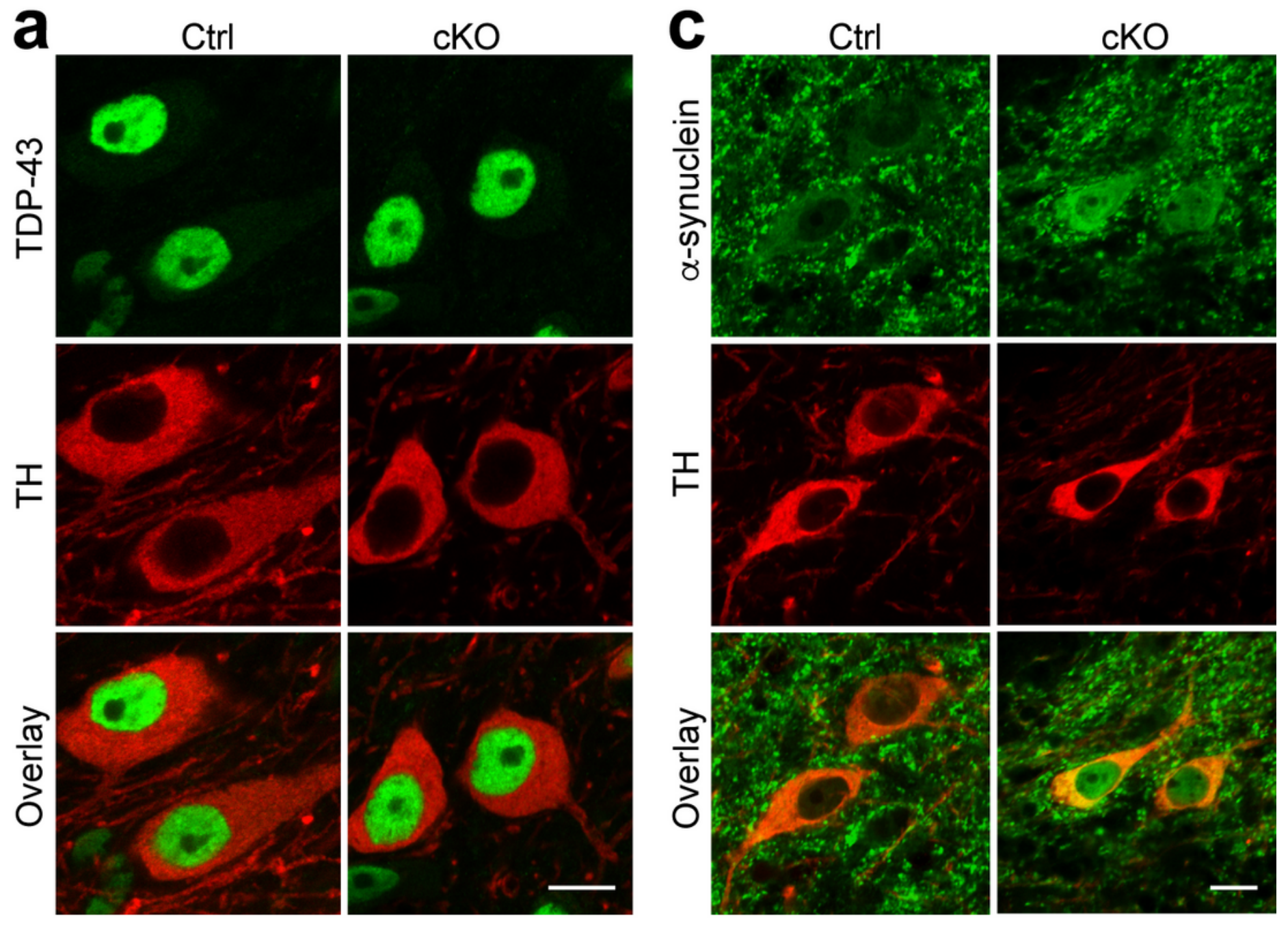

b

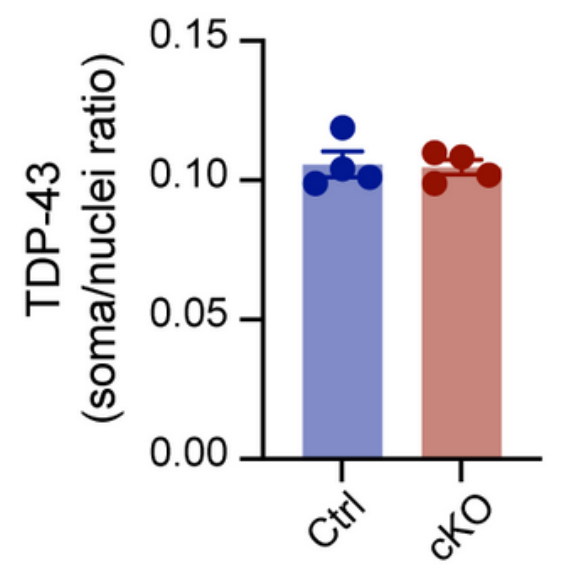

d

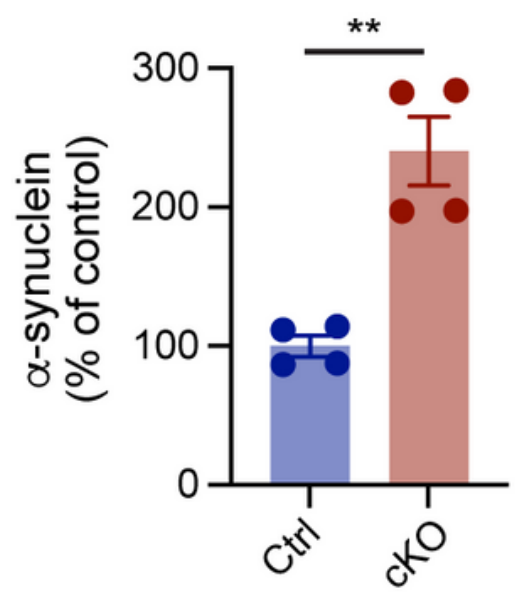

Figure 4

Abnormal accumulation of a-synuclein but not TDP-43 in the soma of cKO DNAs. (a) Immunofluorescent images show the staining of TDP-43 (green) and TH (red) in midbrain coronal sections of 24-month-old Ctrl and cKO mice. Scale bar:20 $\mu \mathrm{m}$. (b) Bar graph quantifies the ratio of soma/nuclei TDP-43 in the DANs $(n=4$ animals per genotype and $\geq 30$ neurons per animal). Data were presented as mean \pm SEM.

Unpaired t-test, $p=0.8547$. (c) Immunofluorescent images show the staining of a-synuclein (green) and TH 
(red) in midbrain coronal sections of 24-month-old Ctrl and cKO mice. Scale bar: $20 \mu \mathrm{m}$. (d) Bar graph quantifies the increase of somatic and nuclear a-synuclein in the cKO DANs compared to the Ctrl ones (n $=4$ animals per genotype and $\geq 30$ neurons per animal). Data were presented as mean \pm SEM. Unpaired t-test, ${ }^{* *} p=0.0016$.

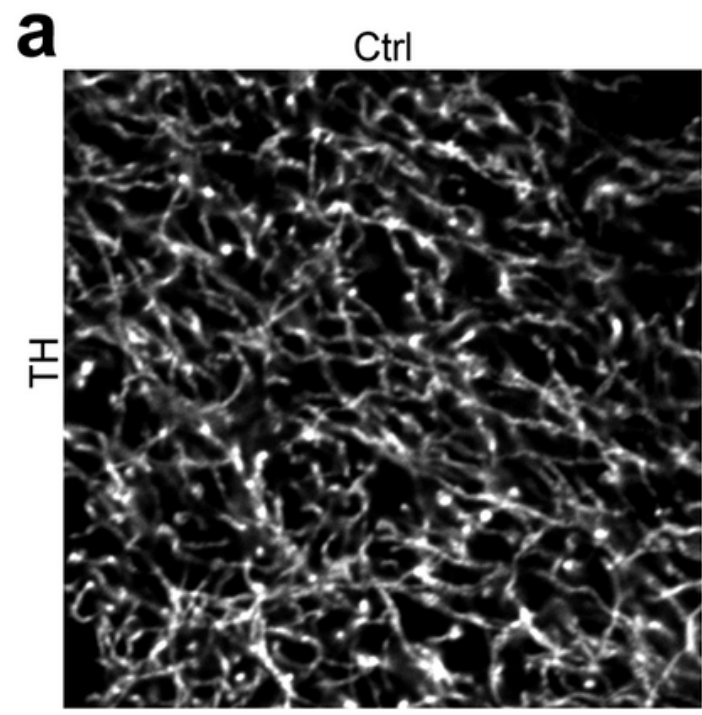

C
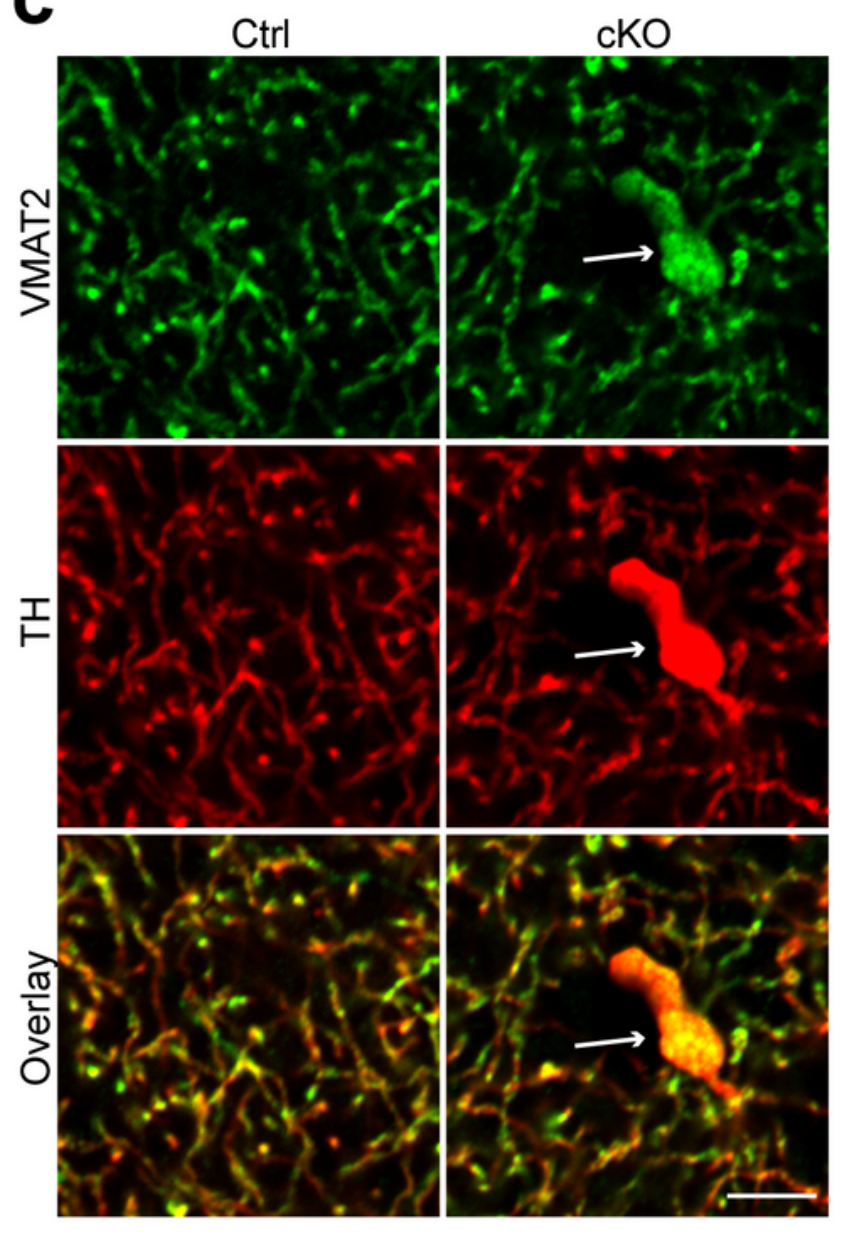

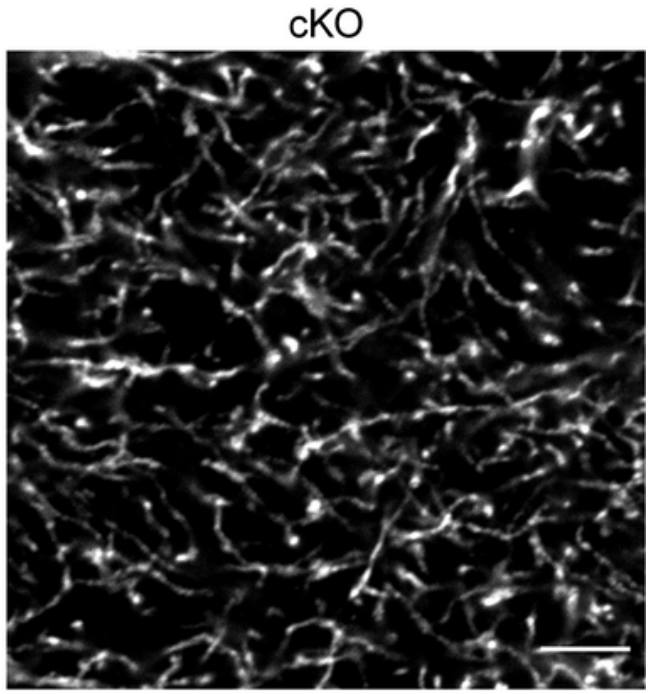

b

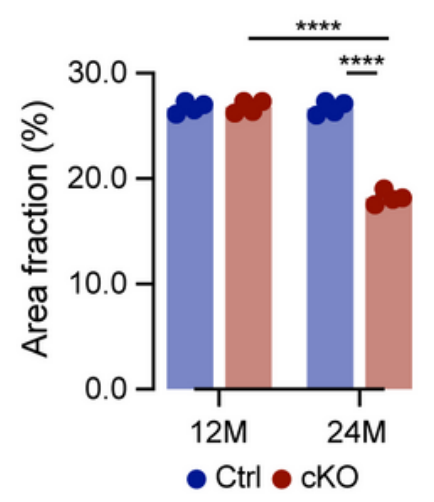

d

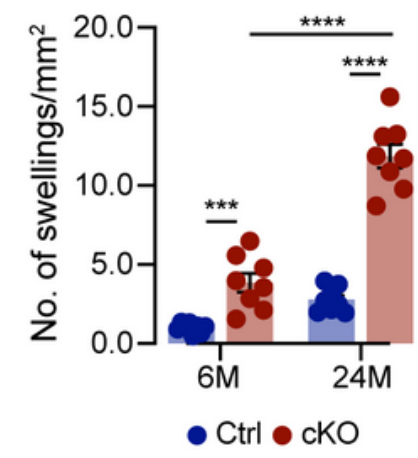

Figure 5 
Progressive degeneration of dopaminergic axon terminals in the striatum of cKO mice. (a) Representative images show TH staining in the dorsal striatum coronal sections of 24-month-old Ctrl and cKO mice. Scale bar: $20 \mathrm{~mm}$. (b) Bar graph estimates the density of TH-positive axon terminals in dorsal striatum of 12- and 24-month-old Ctrl and cKO mice ( $\mathrm{n}=4$ animals per genotype and 10 sections per animal). Data were presented as mean \pm SEM. Unpaired t-test, ${ }^{* \star \star \star} p<0.0001$ (24M). (c) Immunofluorescent images show the staining of VMAT2 (green) and TH (red) in the striatum coronal sections of 6-month-old Ctrl and cKO mice. Scale bar: $20 \mu \mathrm{m}$. Arrows point to a large swelling of VMAT2- and TH-positive dopaminergic axon segment. (d) Bar graph quantifies the number of dopaminergic axonal swellings in dorsal striatum of 6 - and 24-month-old Ctrl and cKO mice ( $\mathrm{n}=8$ animals per genotype and 5 sections per animal). Data were presented as mean \pm SEM. Unpaired t-test, ${ }^{* \star *} p=0.0004,{ }^{* \star \star *} p<0.0001$. 

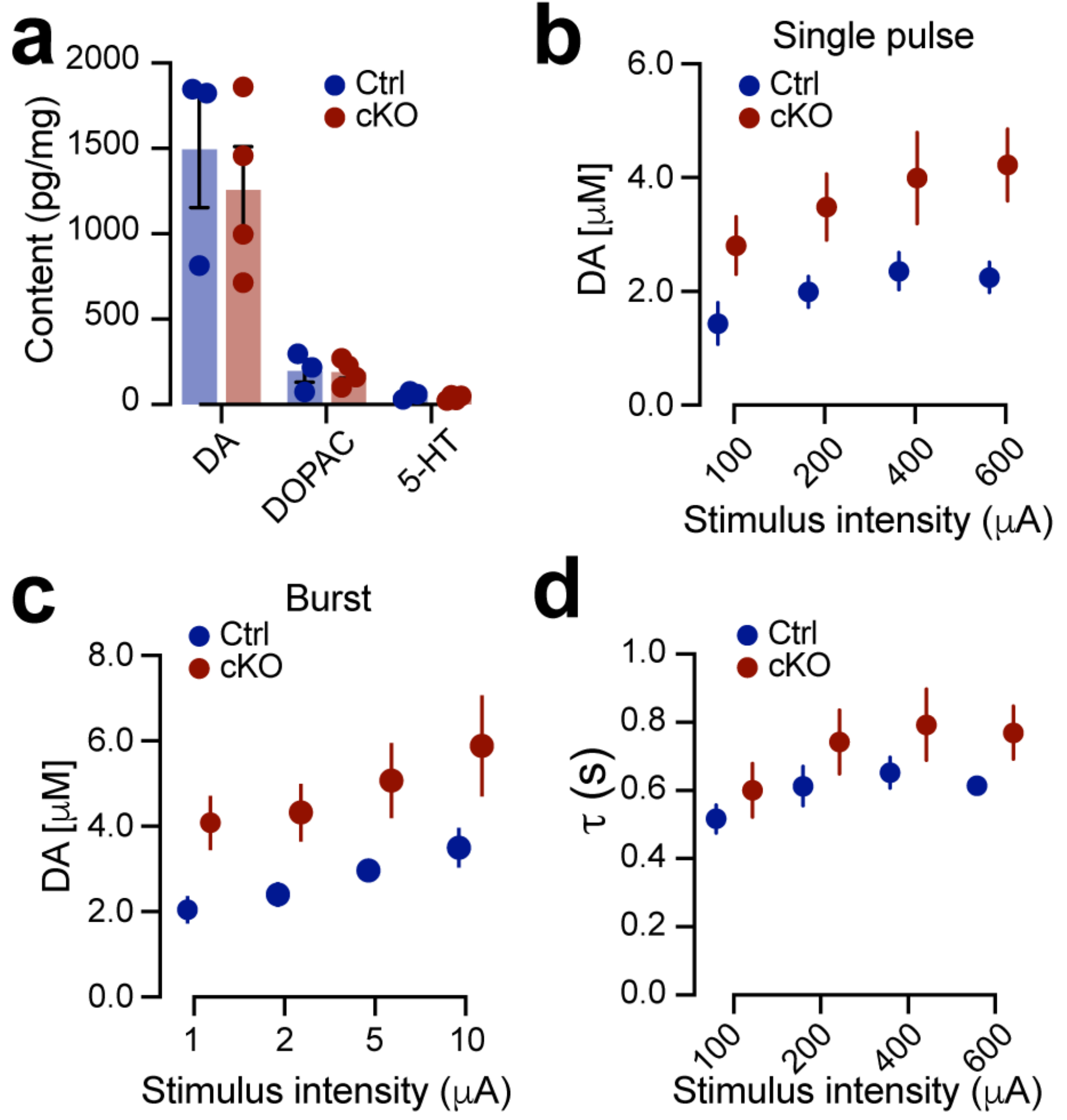

Figure 6

Alterations of dopamine release and uptake in the dorsal striatum of adult cKO mice. (a) HPLC measures the content of dopamine (DA), DOPAC and 5-HT in the dorsal striatum of 12-month-old Ctrl $(n=3)$ and cKO mice $(n=4)$. Data were presented as mean \pm SEM. Unpaired t-test, no significant difference. (b, c) FSCV quantifies the peak evoked dopamine release in response to single-pulse (b) or burst (c) electrical stimulation in the dorsal striatum of 12-month-old Ctrl and cKO mice $(n=3$ animals per genotype and 3 
sections per animal). Data were presented as mean \pm SEM. Two-way ANOVA, genotype factor: $F_{(1,16)}=$ $25.11,{ }^{\star \star *} p=0.0001$ in (b); $F_{(1,16)}=23.09,{ }^{\star \star *} p=0.0002$ in (c). (d) The time constant of slope decay (t) following different single-pulse stimulus intensity in the dorsal striatum of 12-month-old $\mathrm{Ctrl}(\mathrm{n}=3$ animals per genotype and 3 sections per animal) and cKO mice $(n=4$ animals per genotype and 3 sections per animal). Data were presented as mean \pm SEM. Two-way ANOVA, genotype factor: $F_{(1,20)}=$ $5.228,{ }^{*} p=0.03$.

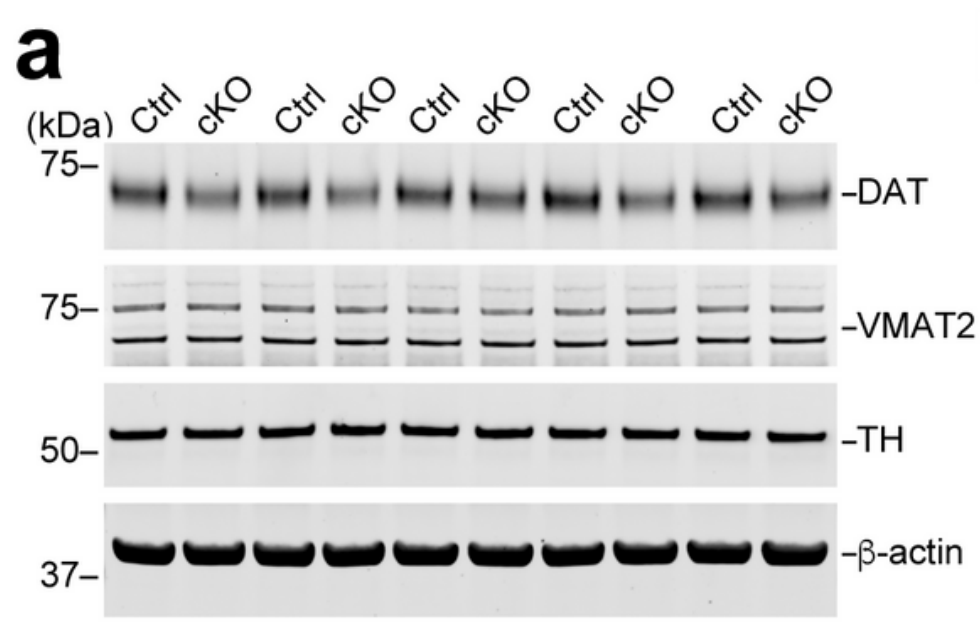

b

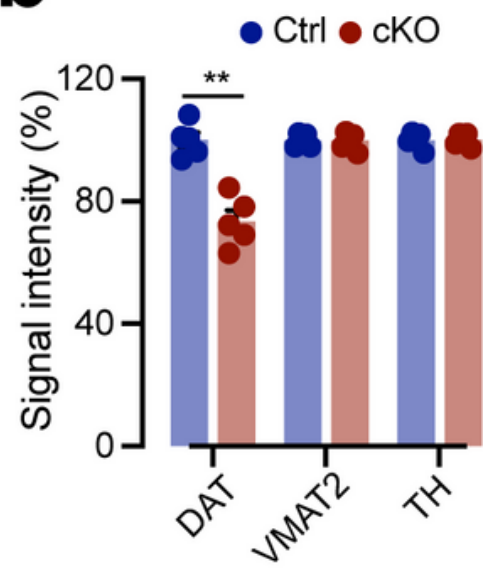

C

Ctrl
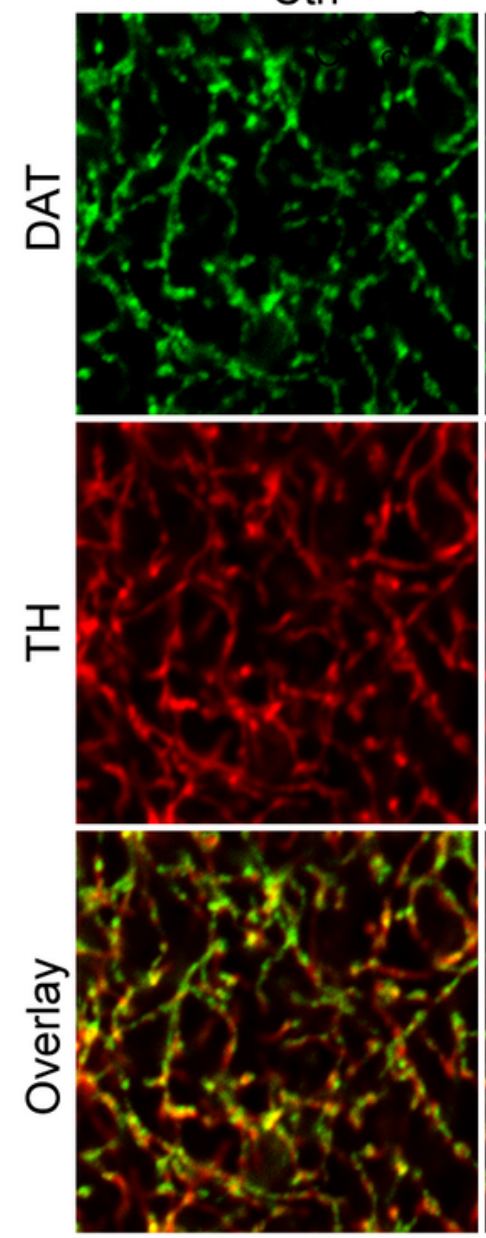

CKO
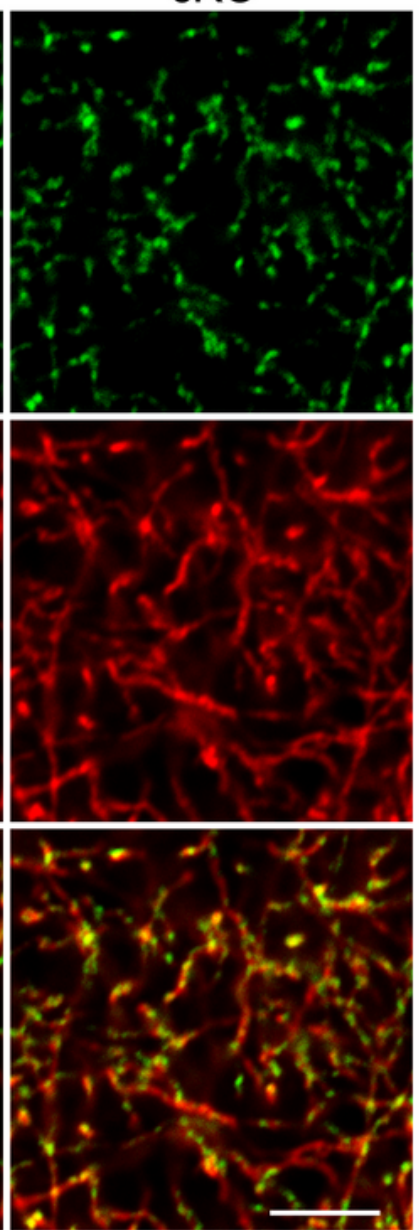

d

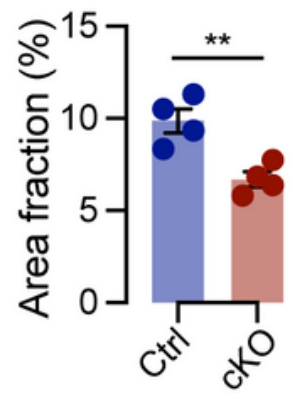

$\mathbf{e}$

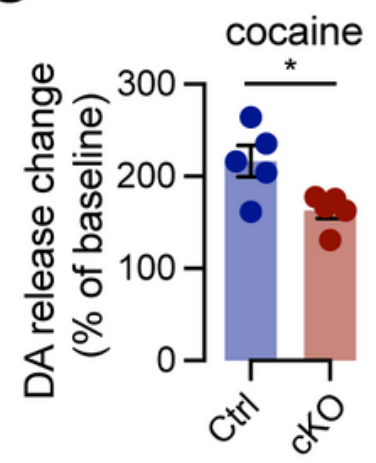




\section{Figure 7}

Reduction of dopamine transporter (DAT) expression in the striatum of young cKO mice. (a) Western blots show the expression of DAT, VMAT2 and TH in the striatum homogenates of 6-month-old Ctrl and cKO mice. b-actin was used as loading control. (b) Bar graph estimates the levels of DAT, VMAT2 and TH expression normalized against $b$-actin $(n=5$ per genotype). Data were presented as mean \pm SEM. Unpaired t-test, ${ }^{\star \star} p=0.0030$. (c) Immunofluorescent images show the staining of DAT (green) and TH (red) in the dorsal striatum coronal sections of 6-month-old Ctrl and cKO mice. Scale bar: $20 \mu \mathrm{m}$. (d) Bar graph estimates the area fraction of DAT-positive dopaminergic axon terminals in dorsal striatum of 6month-old Ctrl and cKO mice ( $\mathrm{n}=4$ animals per genotype and 5 sections per animal). Data were presented as mean \pm SEM. Unpaired t-test, ${ }^{* *} p=0.0060$. (e) FSCV measures the percentage changes of peak evoked dopamine release after DAT blockage by cocaine in the dorsal striatum of 6-month-old Ctrl and CKO mice ( $\mathrm{n}=5$ animals per genotype and 2 sections per animal). Data were presented as mean \pm SEM. Unpaired t-test, ${ }^{*} p=0.0225$. 


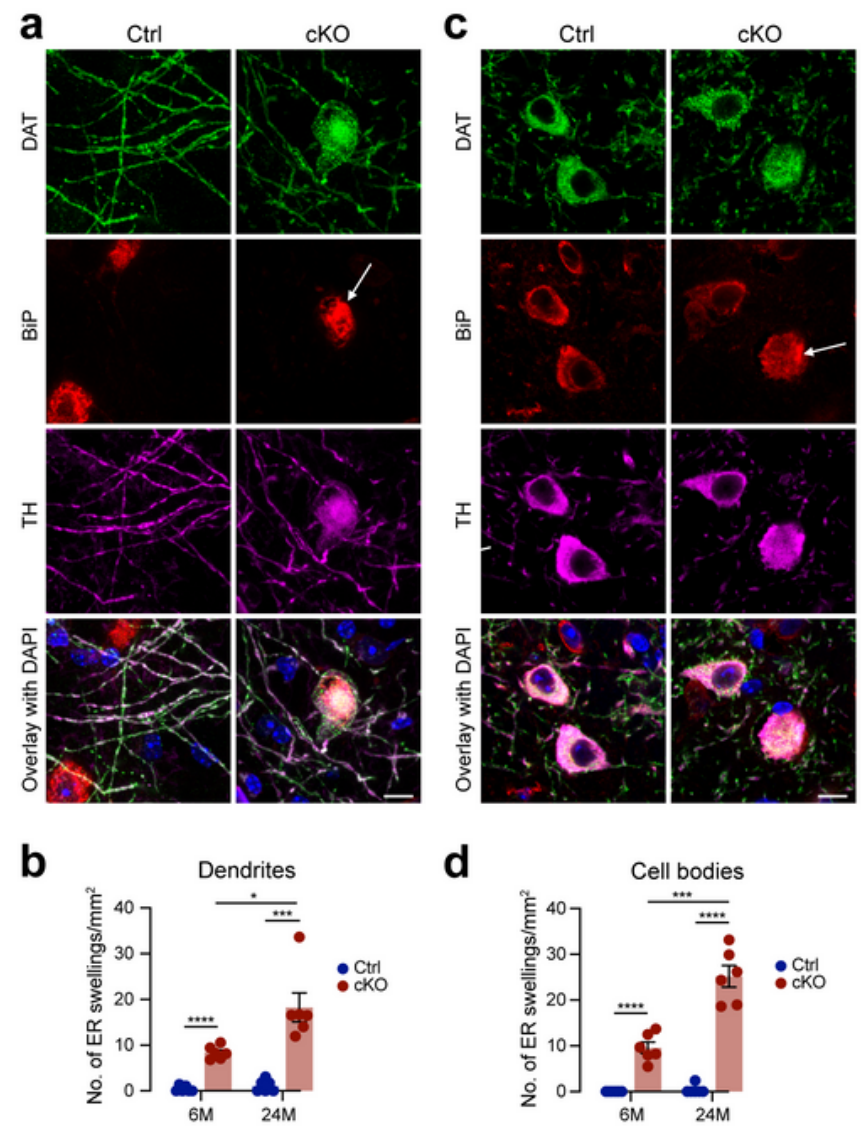

Figure 8

Abnormal ER swellings in midbrain dopaminergic neurons of Dctn1 cKO mice. (a) Fluorescent images show the staining of DAT (green), BiP (red), TH (magenta), and DAPI (blue) in the SNr of 6-month-old Ctrl and cKO mice. Scale bar: $20 \mu \mathrm{m}$. Arrows point to BiP-positive dendritic spheroids. (b) Bar graph quantifies the number of $\mathrm{BiP}$ and $\mathrm{TH}$-positive dendritic spheroids in the SNr of 6- and 24-month-old Ctrl and cKO mice ( $n=6$ animals per genotype and 4 sections per animal). Data were presented as mean \pm SEM. 
Unpaired t-test, ${ }^{*} p=0.0122,{ }^{* \star *} p=0.0003,{ }^{* \star *} p<0.0001$ (24M). (c) Fluorescent images show the staining of DAT (green), BiP (red), TH (magenta), and DAPI (blue) in the SNc of 6-month-old Ctrl and cKO mice.

Scale bar: $20 \mu \mathrm{m}$. Arrow points to a large BiP-positive aggregates in the soma of TH-positive dopaminergic soma. (d) Bar graph quantifies the number of DANs possessing large BiP-positive aggregates in the midbrain of 6 - and 24-month-old Ctrl and cKO mice ( $\mathrm{n}=6$ animals per genotype and 4 sections per animal). Data were presented as mean \pm SEM. Unpaired t-test, ${ }^{\star \star \star} p=0.0002,{ }^{\star \star \star \star} p<0.0001$.
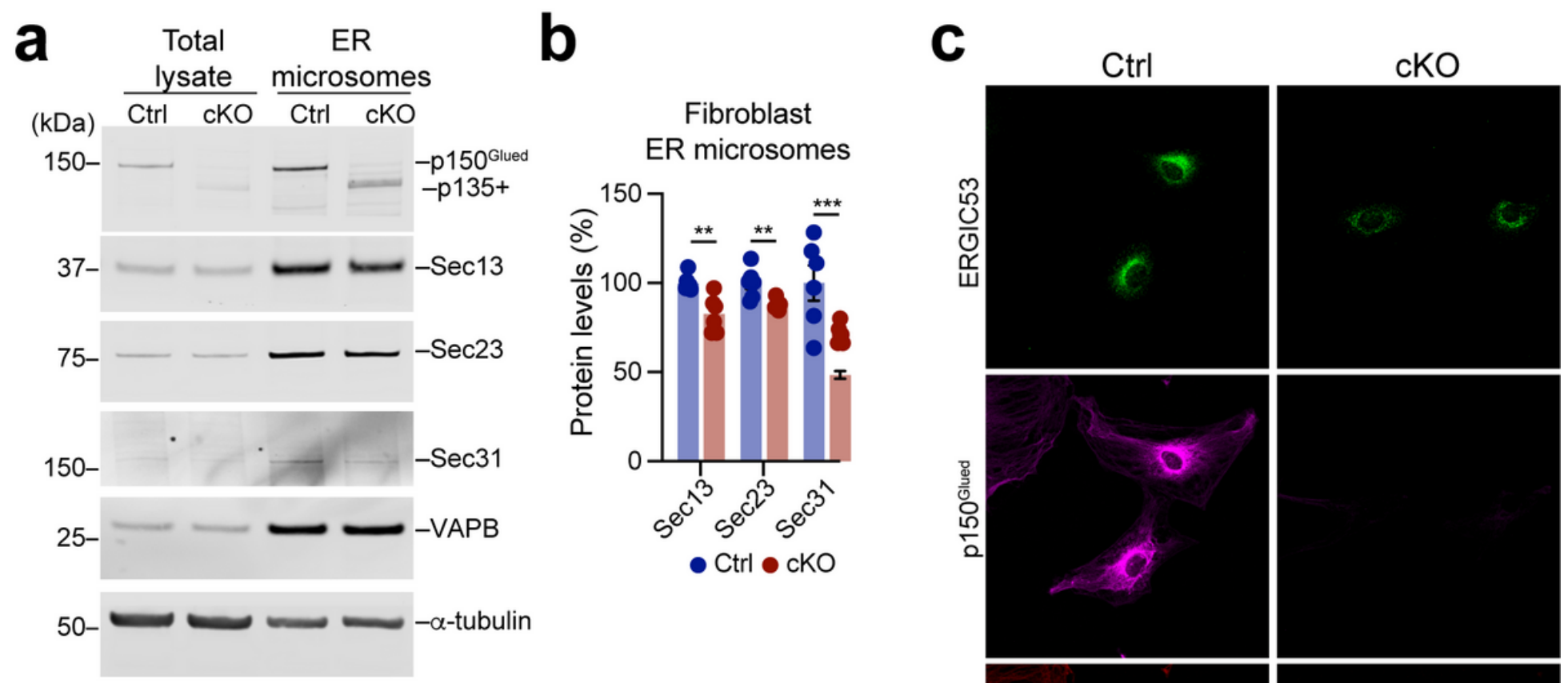

d

Total ER lysate microsomes

(kDa) Ctrl icKO Ctrl icKO

$150---p 150^{\text {Glued }}$

$150-\square=\ldots$
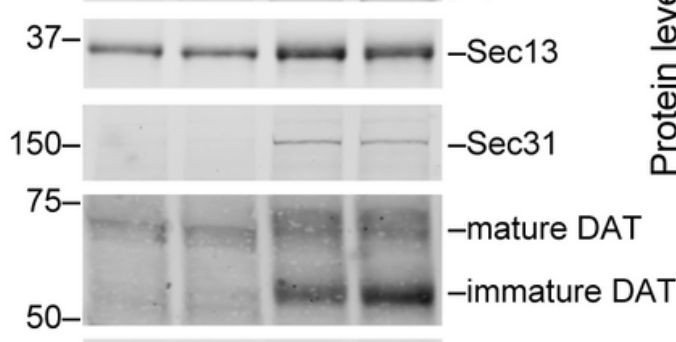

$75-$

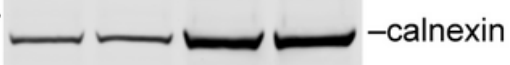

$25-\longrightarrow-\mathrm{VAPB}$

$37-\square=-\beta$-actin
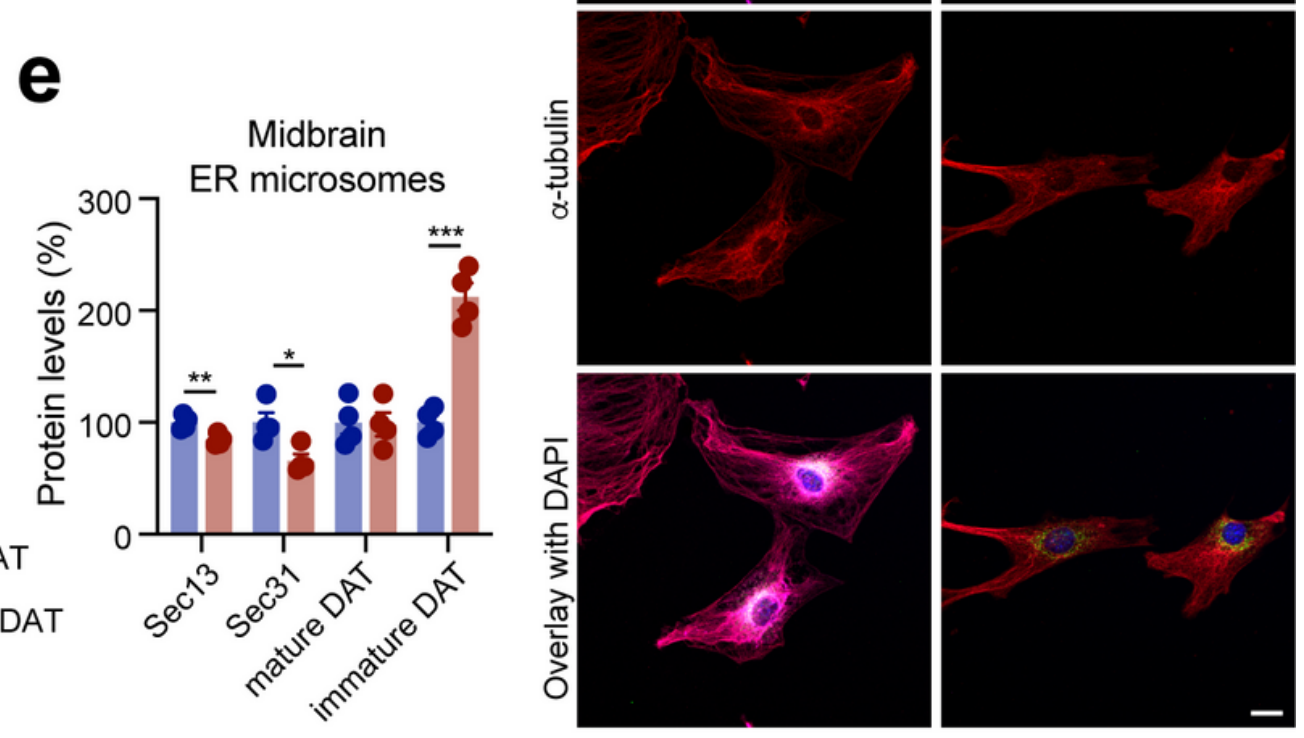

$\bullet$ Ctrl • icKO

Figure 9

P150 Glued deficiency leads to reduction of COPII proteins in ER microsomes and impairment of ERdependent protein export. (a) Western blots show the protein levels of COPII subunits, including Sec13, 
Sec23 and Sec31, in the total lysate and ER microsome fraction of Ctrl and cKO fibroblasts at 14 DIV. VAPB was used as a marker of ER microsome fraction. Tubulin was used as loading control. (b) Bar graph quantifies the levels of COPIl subunits, normalized against VAPB $(n=6$ independent cultures per genotype). Data were presented as mean \pm SEM. Unpaired t-test, ${ }^{* \star} p=0.0032(\operatorname{Sec} 13),{ }^{* \star} p=0.0097$ (Sec23), ${ }^{* \star} p=0.0005$ (Sec31). (c) Fluorescent images show the staining of ERGIC (green), p150 Glued (magenta), a-tubulin (red) in Ctrl and cKO fibroblasts at 14 DIV. Scale bar: $20 \mu \mathrm{m}$. (d) Western blots show the protein levels of $\mathrm{p} 150^{\text {Glued }}$, Sec13, Sec31, mature and immature DAT in the total lysate and ER microsome fraction isolated from midbrain of Ctrl and icKO mice. Calnexin and VAPB were used as a marker of ER microsome fraction. Actin was used as loading control. (e) Bar graph quantifies the levels of COPII subunits and DAT normalized against VAPB in midbrain ER microsome fraction $(n=4$ experiments per genotype). Data were presented as mean \pm SEM. Unpaired t-test. ${ }^{* \star} p=0.009(\operatorname{Sec} 13),{ }^{*} p=0.0187$ (Sec31), ${ }^{\star \star \star} p=0.0002$ (immature DAT). 
a
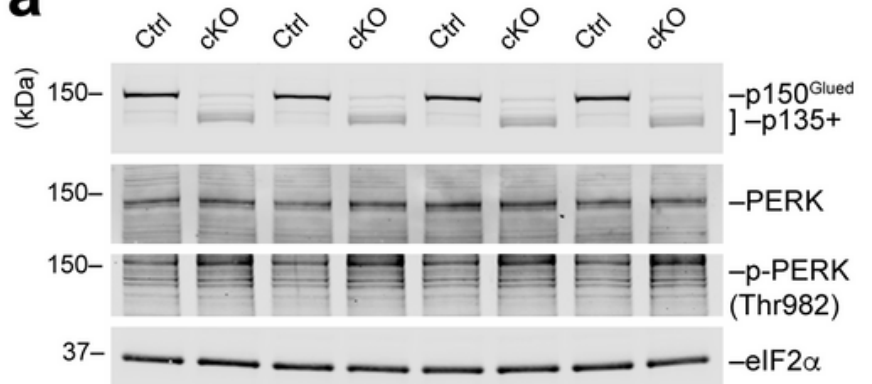

$37--------\begin{aligned} & -\mathrm{p} \text {-elF2 } \alpha \\ & (\text { Ser52) }\end{aligned}$

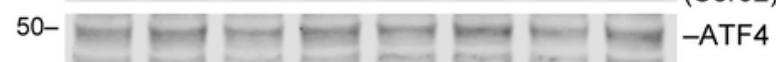

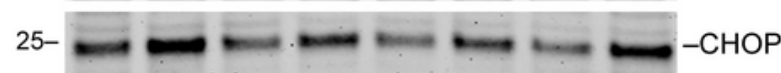

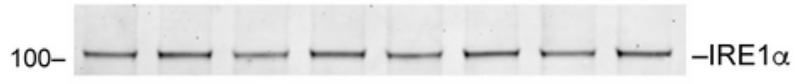

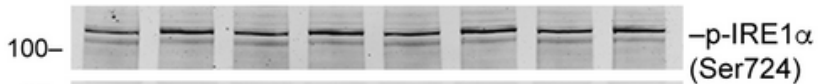

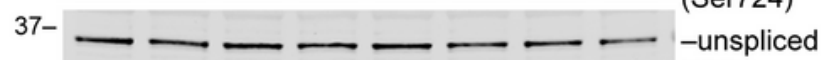

XBP1

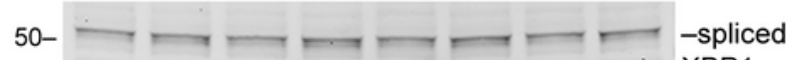

XBP1

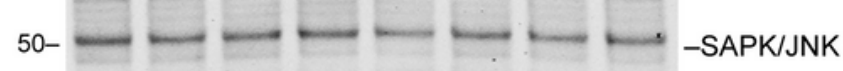

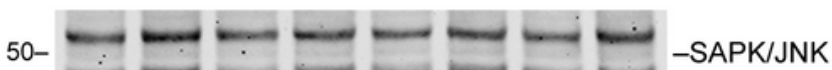

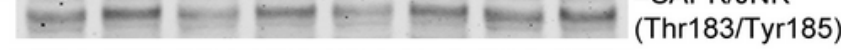

$100-\sqcup \sqcup \sqcup \sqcup \sqcup \sqcup-$-ATF6

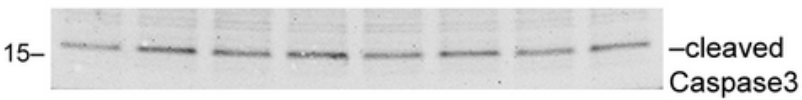

$50-\square=\square-\alpha-$-tubulin

b

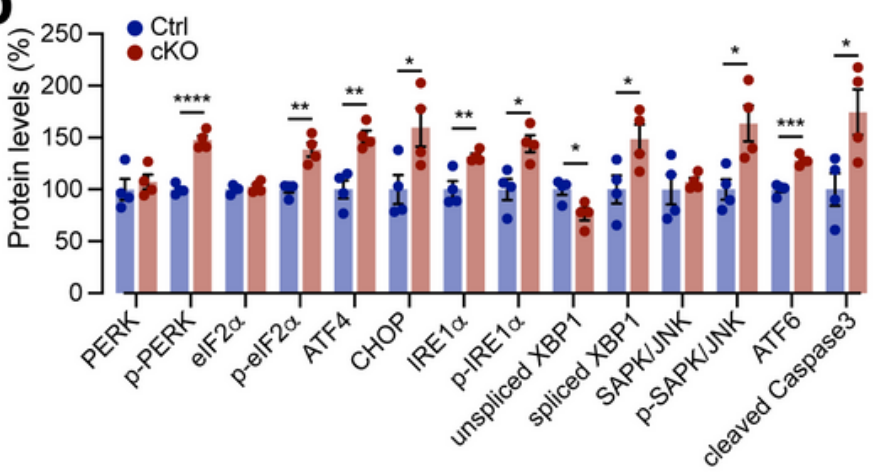

C
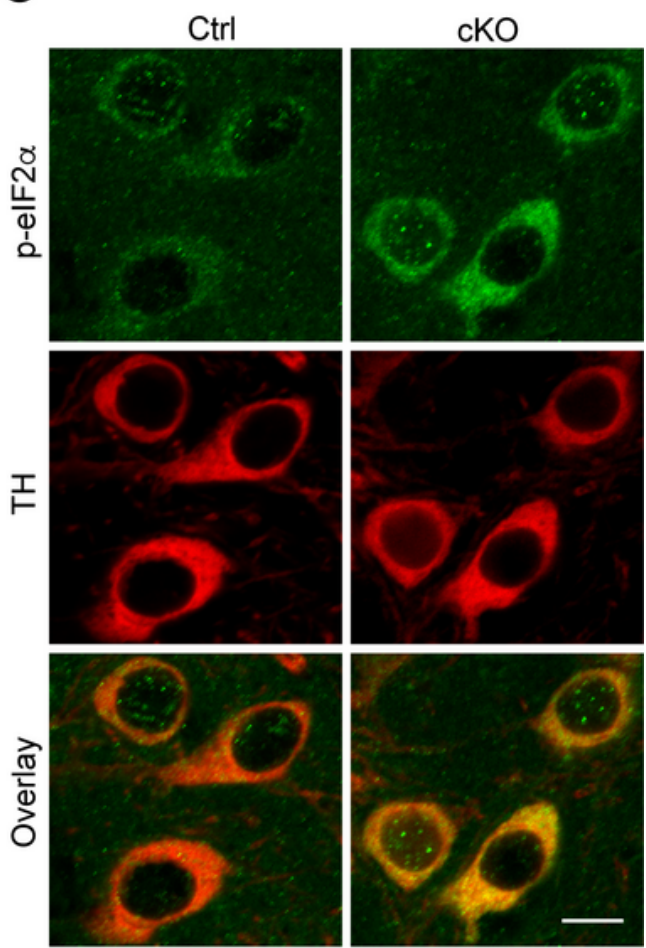

d

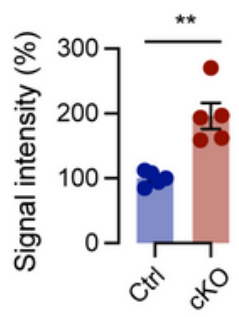

e

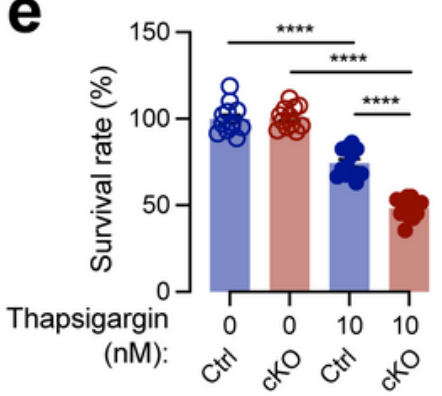

Figure 10

P150 Glued deficiency activates unfolded protein reaction and exacerbates ER stress-induced death of DANs. (a) Western blots show the expression of UPR pathway proteins and activated Caspase 3 in the total lysates of Ctrl and cKO fibroblasts at 14 DIV. Tubulin was used as loading control. (b) Bar graph estimates the levels of protein expression normalized against a-tubulin $(n=4$ independent cultures per genotype). Data were presented as mean \pm SEM. Unpaired t-test. ${ }^{* \star *} p \otimes 0.0001$ ( $p$-PERK), ${ }^{* \star} p=0.0021$ (p- 
elF2a), ${ }^{* *} p=0.0028$ (ATF4), ${ }^{*} p=0.0405$ (CHOP), ${ }^{* *} p=0.0096$ (IRE1a), ${ }^{*} p=0.0141$ (p-IRE1a), ${ }^{*} p=0.0237$ (unspliced XBP1), ${ }^{*} p=0.0451$ (spliced XBP1), ${ }^{*} p=0.0190$ (p-SAPK/JNK), ${ }^{\star \star \star} p=0.0003$ (ATF6), ${ }^{*} p=$ 0.0317 (cleaved Caspase3). (c) Immunofluorescent images show the staining of p-elF2a (Ser52) (green) and TH (red) in the midbrain of 18-month-old Ctrl and cKO mice. Scale bar: $20 \mu \mathrm{m}$. (d) Bar graph quantifies the signal intensity of p-elF2a in the soma of midbrain DANs of 18-month-old Ctrl and cKO mice ( $n=5$ animals per genotype and $\geq 20$ neurons per animal). Data were presented as mean \pm SEM. Unpaired t-test. ${ }^{* *} p=0.0016$. (e) Bar graph shows the survival rate (percentage) of cultured Ctrl and cKO midbrain DANs (14 DIV) treated with 0 or $10 \mathrm{nM}$ thapsigargin for $48 \mathrm{~h}(\mathrm{n}=12$ coverslips per genotype per condition). Data were presented as mean \pm SEM. One-way ANOVA plus Tukey's post hoc test. ${ }^{\star \star \star \star} \mathrm{p}<$ 0.0001 . No difference was found between Ctrl and cKO DANs treated with $0 \mathrm{nM}$ thapsigargin.

\section{Supplementary Files}

This is a list of supplementary files associated with this preprint. Click to download.

- Dctn1suppIMND.docx 